\title{
The Optimal Insurance Policy for the General Fixed Cost of Handling an Indemnity under Rank-Dependent Expected Utility
}

\author{
Liurui Deng \\ College of Economics and Management, Hunan Normal University, Changsha 410081, China \\ Correspondence should be addressed to Liurui Deng; purplerosed@yahoo.com
}

Received 20 July 2015; Revised 21 October 2015; Accepted 22 October 2015

Academic Editor: Walter Briec

Copyright (C) 2015 Liurui Deng. This is an open access article distributed under the Creative Commons Attribution License, which permits unrestricted use, distribution, and reproduction in any medium, provided the original work is properly cited.

\begin{abstract}
Based on Bernard et al.'s research, we focus on the Pareto optimal insurance design with the insured's Rank-Dependent Expected Utility (RDEU). Compared with their previous work, our novelties are the more general fixed cost function of the insurer and the discussion of adverse selection and moral hazard. In particular, Bernard et al. only consider the case in which the fixed cost function of handling an indemnity is the linear function. However, the fixed cost function is not just linear functions in real insurance market. So, we explore the more general fixed cost function including both the linear and nonlinear functions. On the other hand, we consider adverse selection and moral hazard which are involved by Bernard et al. Leading adverse selection and moral hazard into our research makes our results more practical and meaningful. Moreover, we provide an insight into the sensitivity of an optimal solution for the insured's initial wealth and the parameters related to the fixed cost function of handling an indemnity. We further compare the two different utility functions of the insured in terms of influence of optimal policy analysis.
\end{abstract}

\section{Introduction}

In an economic system, both companies and individuals have to face uncertainty in the future. In such a situation, the redistribution of risk among the firms and individuals is facilitated. Usually, persons use insurance policies for reallocating risk. In the late 1960s, Mossin et al. initiated the insurance decision analysis [1-3], where they focused on relationally purchasing insurance from the viewpoint of an individual who has to face a specific risk, given his preference structure and wealth level. Their appealing research builds on the assumption that the insurance policy is exogenously specified. However, Borch [4] and Arrow [5, 6] object to Mossin's opinion and firmly believe that the insurance contract is not exogenous. Sappington [7] provides a complete justification of principalagent modeling for the insurance problem. He sets up the basic framework and the key model of game relationship between the principal and the agent. Furthermore, he builds a monitoring mechanism based on the prisoner's dilemma and builds an incentive mechanism by competition.

In contrast with Sappington's principal-agent model, the insured corresponds to the agent and the insurer corresponds to the principal. Actually, our problem is different from his.
In his model, the agent first decides whether to accept or reject the contract. After the agent signs the contract, he can change his own expected utility by choosing the efficient level of effort. In our model, the agent first determines whether to accept or reject the contract too. But, once the agent agrees to and signs the insurance contract, he cannot change his own value function which is only determined by the random loss $x$.

Although there are obvious differences between our models, our research is consistent with his basic framework. We can make a close link with his research. (1) As an agent, the insured should pay the upfront premium $Q$ for sharing the loss with the insurer. This is as if the insured has to pay the "franchise fee" $k$ for the right to work for the principal; (2) in his research, the agent will accept the contract offered by the principal if and only if the subsequent self-interested behavior under the terms of the contract provides the agent with a level of expected utility that exceeds his reservation level, $\bar{U}$. The insured's criterion of buying or not buying the insurance is

$$
U^{R}\left(w_{0}-x-Q+I(x)\right) \geq U\left(w_{0}-x\right),
$$


where $U\left(w_{0}-x\right)$ is equal to $\bar{U}$ in his paper; (3) in his paper, this contract promises payments, $P$, to the agent that are precisely the principal's valuation of the agent's performance minus some fixed constant $k$. Formally, $P(X)=V(X)-k$. This is consistent with the insurer's safety loading. In particular, the insurer will price the indemnity in such a way that

$$
Q \geq E[I(x)+C(I(x))] .
$$

In a competitive insurance market, we can understand $E[I(x)+C(I(x))]$ as the minimum price of the indemnity $I(X)$ for a risk-neutral insurer to participate in the business.

Beyond insurance policy analysis, expected utility theory (EUT) has an underlying assumption that the decision maker is rational and uniformly risk averse, only considering the objective probability rather than the subjective probability [8]. In reality, however, various decision makers' behaviors deviate from the implications of expected utility. Substantial experimental and empirical evidence identifies that expected utility theory is incompatible with human observed behavior. The abundant paradoxes lead to the development of a more realistic theory. It is dominant in prominent paradigms that Tversky and Kahneman propose prospect theory (PT) [9]. Later, they develop their prospect theory to cumulated prospect theory (CPT) since CPT is consistent with the firstorder stochastic dominance [10]. In the context of CPT, they incorporate human emotion into their investigation.

The incentive for the optimal decision, especially to the optimal insurance policy, is extensively accepted. Quiggin [11] revolutionized the classical expected utility theory (EUT) by rank-dependent utility (RDU). His framework provides the theoretical background for the essentiality of design of insurance contracts. In 2000, Chateauneuf et al. [12] presented the Choquet expected utility framework and gave some results in the RDU framework as a special case. The Pareto efficient insurance contracts under RDU are illustrated in Carlier and Dana's work [13, 14]. Dana and Scarsini [15] mention optimal risk sharing with background risk and briefly identify the case of RDU. He and Zhou [16, 17] emphasize the optimal insurance contract when the distortion is convex. Subsequently, Carlier and Dana [18] investigate two-person efficient risk-sharing problems about concave law-invariant utilities and give a characterized result which is valid for any RDU. Carlier and Dana [19] derive the optimal contingent claim for two significant decision frameworks, the RDU and the CP. However, these papers do not obtain the explicit solution while Bernard et al. [20] do so, when the utility function of the insured is concave.

Bernard et al.'s work inspires us to explore the optimal insurance design under Rank-Dependent Expected Utility. Compared with their research, our novelties are the generalization of the cost function and the discussion of adverse selection and moral hazard.

In detail, the key contribution of Bernard et al. is to get the explicit solution of the optimal insurance contract. But this result implicitly relies on the concavity of the extreme point function $H_{\lambda}(z)$ (equation (8) in the paper of Bernard et al. [20]). However, $H_{\lambda}(z)$ is concave, only when the fixed cost of handling the indemnity $C(I(x))$ is a linear function of $I$. So they only discuss the case in which the fixed cost function is linear; that is, $C(I(x))=\rho I(x)$. However, in a real insurance market, the different insurers have various cost functions including linear functions and other nonlinear functions. So, we pay attention to the more general cost functions to make the results more practical. Generalizing the fixed cost of handling the indemnity brings us a divers obstacle from Bernard and Zhou's work: we are not sure about the convexity or concavity of the extreme point function $J(z)$ ((32) in Section 3). In other words, this general cost function results in uncertain monotonicity of the extreme point function $J(z)$ which is different from the proposition of the extreme point function $H_{\lambda}(z)$ in Bernard and Zhou's article. Further, this uncertainty of monotonicity makes us have to discuss the different monotonic intervals of $J(z)$ and five different relationships between $J(z), F_{x}^{-1}(z)$, and $k(z)$ (see Figure 3) while Bernard and Zhou only need to consider one monotonic interval of $J(z)$ and one relationship between $J(z), F_{x}^{-1}(z)$, and $k(z)$ (see Figure 3). Although the various relationships lead to complicated discussion, these relationships make our novel results more general. In fact, Case 1 in Figure 3 coincides with Bernard and Zhou's Figure 2. Through discussing five different cases and the different monotonic intervals, we attain the explicit solution which is the general result applying to both the linear cost functions and nonlinear functions.

Another novel contribution is the discussion about two critical issues which are adverse selection and moral hazard while Bernad and Zhou do not involve them. In particular, we use the following bonus-malus system

$$
\begin{aligned}
& Q \\
& = \begin{cases}\delta Q_{0} & \text { if no accident occurred in the previous period } \\
\gamma Q_{0} & \text { if an accident occurred in the previous period }\end{cases}
\end{aligned}
$$

to determine the premium $Q$, where $Q_{0}$ is the premium in the previous period. We can estimate $\delta$ and $\gamma$ by empirical data to decide the premium $Q$. If $Q$ is the premium in the first period and we have no empirical data and $Q_{0}$, we have to decide $Q$ relying on the indexes associated with $Q$, such as age, gender, and occupation. Based on this fixed $Q$, we research the optimal problem for indemnity $I(x)$ under Rank-Dependent Expected Utility. Although we only offer a brief thought of how to decide $Q$ and not carefully research it, this significant thought not only makes close link between these critical issues and our research but also offers the basic framework for further research.

Recently, Dhiab investigates the demand for insurance under the nonexpected utility theory [21]. He applies RankDependent Expected Utility (RDEU) to the insurance contract. In his insurance context, agents behave not only according to their probability distribution but also according to their attitude towards risk.

Although Ben Dhiab's research is similar to mine, there are obvious differences between our researches. The important difference is that we research the insurance problem from different angles. In particular, he researches the optimal insurance contract from the insured's (or agent's) point of 
view, so he only needs to maximize the RDEU of the insured without considering the utility function of an insurer and relative restrictive conditions. However, we study the optimal insurance policy from an insurer's point of view. Thus, we set up the optimal insurance policy subjected to the restrictive condition associated with the utility function of the insurer. Meanwhile, the insurer's utility functions and the optimal solutions change due to different insurers' cost functions $C(I)$. So, our calculations and results are more complicated than his.

Another difference is that he does not present the quantified relationship between the indemnity function $I(x)$ and the random loss $x$ when the optimal insurance policies are partial insurance and overinsurance. However, $I$ reveal the accurately quantified relationship between the indemnity function $I(x)$ and the random loss $x$ in Proposition 9.

Furthermore, there are two main differences on the technological detail. (1) He supposes that the probability weighting function (probability distortions) is always concave or convex. But, the $S$-shape probability weighting function is more reasonable than the concave or convex probability weighting function, because Kahneman and Tversky used sufficient experiments and evidences to extensively demonstrate that not only do people often overweight lowprobability and certain outcomes but also the individual's attitude to the risk always changes. So, we employ the $S$-shape probability weighting function; (2) Dhiab only discusses two states of the nature which are the loss $x$ with probability $p$ and no-loss with probability $1-p$. We explore the more general and complicated case in which the loss $x$ is a random variable on $\left[0, w_{0}\right]$. The general $x$ means that we cannot attain the optimal solution by directly calculating and simply discussing as Dhiab does. So we have to use quantile function for solving the optimal problem.

This paper is organized as follows: In Section 2, we set up critical models. Section 3 explores the optimal solutions. In Section 4, numerical analysis is performed. Section 5 summarizes the conclusions. In Section 6, we introduce the further research. The paper ends with an the Appendix containing the proofs.

\section{The Model}

In this section, we focus on the Pareto optimal insurance contract where the insured has Rank-Dependent Expected Utility [11] preference.

\subsection{The Basic Setting}

2.1.1. The Original Insurance Problem. Let $(\Omega, \mathscr{F}, \mathbb{P})$ be a probability space. An economic agent, called a policyholder or the insured, is endowed with the initial wealth $w_{0}$ and has to face the a nonnegative random loss $x$ with support in $\left[0, w_{0}\right]$. The initial wealth of the insurer is $W_{0}$. The loss $x$ is a random variable with the probability density function $p(x)$. The insured should pay the upfront premium $Q$ for sharing the loss with the insurer. If the insured stands to the loss $x$, the insurer will pay out $I(x)$. We treat $I(x)$ as the indemnity function of the loss or coverage function. The indemnity principle compensates for the insured's loss when an accident happens. According to this rule, the policyholder cannot collect more money than his actual loss. Hence, we assume $0 \leq I(x) \leq x$. The constraint condition implies that if there is no loss there will be no reimbursement. As for the cost of the insurer, we state that it includes two part, the administrative expenses or other expenses and deadweight loss related to the insured and the insurer. So, we suppose that the cost of the insurer consists of fixed and variable components, which depends on the size of the insurance payment. We denote the cost by $C(I(x))$. And assume that $C(0)=c \geq 0, C^{\prime}(I) \geq 0$, and $C^{\prime \prime}(I)=a \geq 0$.

We further suppose that the utility function of the insurer is $v(\cdot)$. It is easy to see that the insurer's final wealth is $W_{0}+$ $Q-I(x)-C(I(x))$. Here, we suppose $v^{\prime}(\cdot)>0$ and $v^{\prime \prime}(\cdot)=0$.

Consider there exit finite states of the world: the wealth level $w_{1}, w_{2}, \ldots, w_{n}$. And further assume that you can assign probabilities to each of these outcomes. You are fairly optimistic about your future, so you assign a probability $p_{i}$ to the wealth level $w_{i}(i=1,2, \ldots, n) \cdot \sum_{i=1}^{n} p_{i}=1$. We call the series of wealth outcomes a prospect $P_{n}$ and represent this situation using the following convenient format:

$$
P_{n}\left(p_{1}, w_{1}, p_{2}, w_{2}, \ldots, p_{n-1}, w_{n-1}, w_{n}\right) .
$$

The value function $V$ represents the preferences in the EU model. For a prospect $P_{n}$, preferences can be represented by a functional such that

$$
V\left(P_{n}\right)=\sum_{i=1}^{n} p_{i} v\left(w_{i}\right),
$$

where $v: \mathbb{R}^{+} \rightarrow \mathbb{R}$ is a utility function, strictly increasing and unique up to an affine positive transformation function. The value function $V$ is linear in probabilities.

It is necessary to mention that when $v^{\prime \prime}(\cdot)=0$, namely, the insurer is risk-neutral, the utility function $v(\cdot)$ has an important proposition that the expected utility function equals the utility of the expected value; that is, $E v(P)=$ $v(E(P))$, where $P$ is a prospect (see [22]). We suppose, for simplicity, that there are only two states of the world: low wealth $w_{l}$ and high wealth $w_{h}$. And further assume that you can assign probabilities to each of these outcomes. You are fairly optimistic about your future, so you assign a probability $p_{1}$ to low wealth $w_{l}$ and a probability $1-p_{1}$ to high wealth $w_{h}$. This situation can be represented by a prospect $P$ as the following convenient format:

$$
P\left(p_{1}, w_{l}, w_{h}\right) .
$$

Then, it is easy to write

$$
\begin{aligned}
E v(P) & =p_{1} v\left(w_{l}\right)+\left(1-p_{1}\right) v\left(w_{h}\right), \\
v(E(P)) & =v\left(p_{1} w_{l}+\left(1-p_{1}\right) w_{h}\right) .
\end{aligned}
$$

Due to a risk-neutral utility function, from Figure 1, we can attain $E v(P)=v(E(P))$.

Generally, for a prospect

$$
P_{n}\left(p_{1}, w_{1}, p_{2}, w_{2}, \ldots, p_{n-1}, w_{n-1}, w_{n}\right),
$$




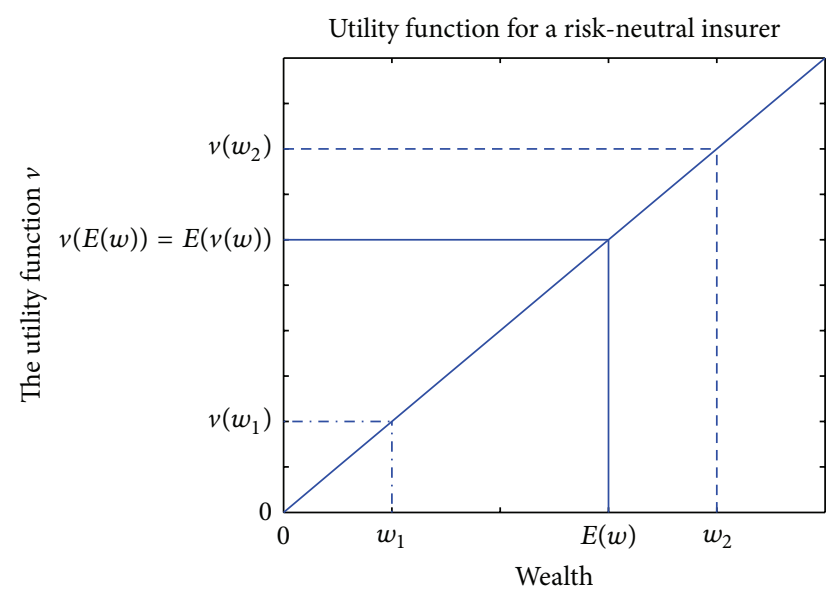

FIGURE 1: Utility function for a risk-neutral insurer.

a similar result is attained:

$$
\begin{aligned}
V\left(P_{n}\right) & =E v\left(P_{n}\right)=\sum_{i=1}^{n} p_{i} v\left(w_{i}\right)=v\left(\sum_{i=1}^{n} p_{i} w_{i}\right) \\
& =v\left(E\left(P_{n}\right)\right) .
\end{aligned}
$$

In the present paper, because the random loss $x$ has finite number of values,

$$
\begin{aligned}
V & \left(W_{0}+Q-I(x)-C(I(x))\right) \\
& =E\left[v\left(W_{0}+Q-I(x)-C(I(x))\right)\right] \\
& =\left(E\left[W_{0}+Q-I(x)-C(I(x))\right]\right)
\end{aligned}
$$

holds.

Meanwhile, our research relies on another essential assumption that the utility function of the insured is $u(\cdot)$ satisfying $u^{\prime}(\cdot) \geq 0$ and $u^{\prime \prime}(\cdot) \leq 0$.

2.1.2. Rank-Dependent Expected Utility. Because the insured prefers Rank-Dependent Expected Utility, we use the decision weights instead of using simple probabilities as expected utility (see [22]).

Definition 1. Let $F_{w}(\cdot)$ be the cumulative distribution function (CDF) of a random variable $w$. The probability distortions are denoted by $G$. One defines the probability weight function (distortions) $G:[0,1] \rightarrow[0,1]:$

$$
\begin{aligned}
G\left(F_{w}(y)\right)=\frac{F_{w}^{\gamma}(y)}{\left(F_{w}^{\gamma}(y)+\left(1-F_{w}^{\gamma}(y)\right)^{\gamma}\right)^{1 / \gamma}}, & \text { with } 0.28<\gamma<1 .
\end{aligned}
$$

Definition 2. Define the RDEU of the insured as (see [12, 23]):

$$
U^{R}(w)=\int_{0}^{+\infty} u(y) d\left(-G\left(1-F_{w}(y)\right)\right),
$$

where $w$ is the final wealth of the insured and $U^{R}(w)$ is the Choquet integral of $u(\cdot)$.
Since the insured's final wealth is $w=w_{0}-x-Q+I(x)$, the RDEU of the insured is as follows:

$$
\begin{aligned}
& U^{R}\left(w_{0}-x-Q+I(x)\right) \\
& \quad=\int_{0}^{+\infty} u(y) d\left(-G\left(1-F_{w_{0}-x-Q+I(x)}(y)\right)\right),
\end{aligned}
$$

where $F_{w_{0}-x-Q+I(x)}$ is the cumulative distribution function (CDF) of $w$ which is the function of the random variable $x$. Thus, at this time, $F_{w}$ is replaced by $F_{w_{0}-x-Q+I(x)}$.

2.1.3. The Optimal Insurance Problem. In order to attract the insured in the business competition, the insurer has to make the insured's gain as much profitable as possible. To explicitly explain the influence of the competition on the optimal problem, we take an example. There two insurers, $A$ and $B$. Because of the different insurer designs of $A$ and $B$, the insured's RDEUs for $A$ is different from his RDEU for $B$. RDEUs are respectively written as $U_{A}^{R}$ and $U_{B}^{R}$. If $U_{A}^{R}>U_{B}^{R}$, the insured prefers the insurer $A$ rather than $B$. Otherwise, the insured is willing to choose the insurer $B$ but not $A$. Since there exit many insurers in a limited insurance market, each insurer tries his best to maximize the RDEU of the insured by designing the optimal insurance contract for attracting more clients.

That is, the insurer will find the optimal solution of $U^{R}$ :

$$
\begin{aligned}
\underset{Q, I(x)}{\operatorname{Max}} U^{R}\left(w_{0}-x-Q+I(x)\right) \\
\quad=\int_{0}^{+\infty} u(y) d\left(-G\left(1-F_{w_{0}-x-Q+I(x)}(y)\right)\right) .
\end{aligned}
$$

On the other hand, as shown in Raviv [24], a necessary condition for the insurer to offer such a policy is

$$
E\left[v\left(W_{0}+Q-I(x)-C(I(x))\right)\right] \geq v\left(W_{0}\right) .
$$

Considering the impact of adverse selection and moral hazard on the insurance contract [25-27], we can use the following bonus-malus system

$$
\begin{aligned}
& Q \\
& = \begin{cases}\delta Q_{0} & \text { if no accident occurred in the previous period } \\
\gamma Q_{0} & \text { if an accident occurred in the previous period }\end{cases}
\end{aligned}
$$

to determine the premium $Q$, where $Q_{0}$ is the premium in the previous period. We can estimate $\delta$ and $\gamma$ by empirical data to decide the premium $Q$. If $Q$ is the premium in the first period and we have no empirical data and $Q_{0}$, we have to decide $Q$ relying on the indexes associated with $Q$, such as age, gender, and occupation. Based on this fixed $Q$, we research the optimal problem of indemnity $I(x)$.

We denote by $\mathscr{I}$ the set of all indemnity functions; that is, $\mathscr{I}:=\left\{I(\cdot) \mid 0 \leq I(x) \leq x, \forall x \in\left[0, w_{0}\right]\right\}$. The optimal problem is as follows. 
Model 1. Consider

$$
\begin{aligned}
\underset{I(\cdot) \in \mathscr{I}}{\operatorname{Max}} & U^{R}\left(w_{0}-x-Q+I(x)\right) \\
& =\int_{0}^{+\infty} u(y) d\left(-G\left(1-F_{w_{0}-x-Q+I(x)}(y)\right)\right)
\end{aligned}
$$

Subject to $E\left[v\left(W_{0}+Q-I(x)-C(I(x))\right)\right] \geq v\left(W_{0}\right)$.

As Raviv has shown, if the insurer is risky-neutral, the insurer will price the indemnity in such a way that

$$
Q \geq E[I(x)+C(I(x))] .
$$

In a competitive insurance market, we can understand $E[I(x)+C(I(x))]$ as the minimum price of the indemnity $I(x)$ for a risk-neutral insurer to participate in the business (typically referred to as the insurer's safety loading).

Noticing that the independent variable $I(x) \in \mathscr{I}$ of the function $C(\cdot)$ is bounded and the function $C(\cdot)$ is continuous, we can say that $C(\cdot)$ is bounded. So, there exits finite $Q$ satisfying restrict condition (18).

So, Model 1 can be rewritten as follows.

Model 2. Consider

$$
\begin{aligned}
\underset{I(\cdot) \in \mathscr{I}}{\operatorname{Max}} & U^{R}\left(w_{0}-x-Q+I(x)\right) \\
& =\int_{0}^{+\infty} u(y) d\left(-G\left(1-F_{w_{0}-x-Q+I(x)}(y)\right)\right)
\end{aligned}
$$

Subject to $Q \geq E[I(x)+C(I(x))]$.

Let $R(x)=x-I(x) . R(x)$ is the part of loss shared by the insured and is the so-called retention function. Let

$$
\mathscr{R}=\left\{R(\cdot) \mid 0 \leq R(x) \leq x, \forall x \in\left[0, w_{0}\right]\right\} .
$$

Then, the above model becomes the following.

Model 3. Consider

$$
\begin{aligned}
\underset{R(x) \in \mathscr{R}}{\operatorname{Max}} & U^{R}\left(w_{0}-Q-R(x)\right) \\
& =\int_{0}^{+\infty} u(y) d\left(-G\left(1-F_{w_{0}-Q-R(x)}(y)\right)\right)
\end{aligned}
$$

Subject to $Q \geq E[x-R(x)+C(x-R(x))]$.

2.2. The Objective Model. Before we analyze this model, we introduce some indispensable assumption and lemmas.

Assumption 3 (see [28]). The loss $x$ has no atom; that is, the cumulative distribution function $F(x)$ of $x$ is the continuous function. Accordingly, its quantile function $F_{x}^{-1}:(0,1) \rightarrow$ $R_{+}$is continuous.

Assumption 4 (see [22]). The probability weighting function $G(\cdot):[0,1] \rightarrow[0,1]$ and satisfies $G(0)=0, G(1)=1, G^{\prime}(\cdot) \geq$ 0 , and

$$
\begin{array}{ll}
G^{\prime \prime}(z) \leq 0 & \text { if } z \in\left[0, z_{0}\right] \\
G^{\prime \prime}(z) \geq 0 & \text { if } z \in\left(z_{0}, 1\right] .
\end{array}
$$

Lemma 5. Suppose $A$ is a constant. $g(x)$ is a random variable with the probability density function $f(\cdot)$ and the cumulative distribution function $F_{g(x)}(\cdot)$. The according quantile function $F_{g(x)}^{-1}$ satisfies that

$$
F_{A-g(x)}^{-1}(z)=A-F_{g(x)}^{-1}(1-z)
$$

The proof is seen in Appendix A.

Lemma 6 (see [28]). With Assumption 3, if $R(x)$ is a feasible solution of Model 3, then $\bar{R}(x)=F_{R(x)}^{-1}\left(F_{x}(x)\right)$ is also feasible with respect to Model 3 and $\bar{R}(x)$ has the same law as $R(x)$.

From Lemmas 5 and 6, we can write the model as follows.

Model 4. Consider

$$
\begin{aligned}
\underset{k(z)}{\operatorname{Max}} & \int_{0}^{1} u\left(w_{0}-Q-k(z)\right) G^{\prime}(z) d z \\
\text { Subject to } & 0 \leq k(z) \leq F_{x}^{-1}(z), \quad 0<z<1, \\
& k \in \mathscr{K}, \\
& Q \geq E[x-k(z)+C(x-k(z))] .
\end{aligned}
$$

Here, $k(z)$ is a quantile function. $\mathscr{K}$ represents the set of all quantile functions. That is, $\mathscr{K}=\{k:(0,1) \rightarrow \mathbb{R} \mid$ $k(\cdot)$ is nondecreasing and left-continuous\}.

Detailedly, form Lemma 5, we can transform RDEU of the insured into

$$
\begin{aligned}
U^{R} & \left(w_{0}-Q-R(x)\right) \\
& =\int_{0}^{+\infty} u(x) d\left(-G\left(1-F_{w_{0}-Q-R(x)}(x)\right)\right) \\
& =\int_{0}^{1} u\left(F_{w_{0}-Q-R(x)}^{-1}(z)\right) G^{\prime}(1-z) d z \\
& =\int_{0}^{1} u\left(w_{0}-Q-F_{R(x)}^{-1}(1-z)\right) G^{\prime}(1-z) d z \\
& =-\int_{1}^{0} u\left(w_{0}-Q-F_{R(x)}^{-1}(z)\right) G^{\prime}(z) d z \\
& =\int_{0}^{1} u\left(w_{0}-Q-F_{R(x)}^{-1}(z)\right) G^{\prime}(z) d z \\
& =\int_{0}^{1} u\left(w_{0}-Q-\bar{R}(x)\right) G^{\prime}(z) d z .
\end{aligned}
$$

Let

$$
k(z)=F_{R(x)}^{-1}(z)=F_{R(x)}^{-1}\left(F_{x}(x)\right)=\bar{R}(x) .
$$

Recalling Lemma 6, we can demonstrate that $k(z)$ satisfies the constraints in Model 4.

With Lagrange dual method, we can obtain the auxiliary problem. 
Model 5. Consider

$$
\begin{aligned}
\operatorname{Max}_{k(z)} & U_{\lambda}(k(z), z)=\int_{0}^{1} u\left(w_{0}-Q-k(z)\right) G^{\prime}(z)+\lambda k(z)-\lambda C\left(F_{x}^{-1}(z)-k(z)\right) d z+\lambda(Q-E x) \\
\text { Subject to } & 0 \leq k(z) \leq F_{x}^{-1}(z), \quad 0<z<1 \\
& k \in \mathscr{K} .
\end{aligned}
$$

\section{The Results}

3.1. The Piecewise Optimal Solution. Now, we only consider the maximal value of

$$
\begin{gathered}
u\left(w_{0}-Q-k(z)\right) G^{\prime}(z)+\lambda k(z) \\
-\lambda C\left(F_{x}^{-1}(z)-k(z)\right) .
\end{gathered}
$$

Set

$$
\begin{gathered}
\frac{\partial}{\partial k(z)}\left[u\left(w_{0}-Q-k(z)\right) G^{\prime}(z)+\lambda k(z)\right. \\
\left.-\lambda C\left(F_{x}^{-1}(z)-k(z)\right)\right]=-u^{\prime}\left(w_{0}-Q-k(z)\right) \\
\cdot G^{\prime}(z)+\lambda\left(1+C^{\prime}\left(F_{x}^{-1}(z)-k(z)\right)\right)=0 .
\end{gathered}
$$

Observing Taylor expansions of $u^{\prime}(\cdot)$ and $C^{\prime}(\cdot)$, we have

$$
\begin{aligned}
u^{\prime}\left(w_{0}-Q-k(z)\right) \approx & u^{\prime}\left(w_{0}-Q\right) \\
& -u^{\prime \prime}\left(w_{0}-Q\right) k(z), \\
C^{\prime}\left(F_{x}^{-1}(z)-k(z)\right)= & C^{\prime}\left(F_{x}^{-1}(z)\right) \\
& -C^{\prime \prime}\left(F_{x}^{-1}(z)\right) k(z) .
\end{aligned}
$$

The above results are applied to (29); it is shown that

$$
\begin{aligned}
& -\left(u^{\prime}\left(w_{0}-Q\right)-u^{\prime \prime}\left(w_{0}-Q\right) k(z)\right) G^{\prime}(z) \\
& \quad+\lambda\left(1+C^{\prime}\left(F_{x}^{-1}(z)\right)-C^{\prime \prime}\left(F_{x}^{-1}(z)\right) k(z)\right) \approx 0 .
\end{aligned}
$$

Then, not considering the other constraints in Model 5, we can get the approximate optimal solution:

$$
J(z)=\frac{\lambda\left(1+C^{\prime}\left(F_{x}^{-1}(z)\right)\right)-u^{\prime}\left(w_{0}-Q\right) G^{\prime}(z)}{\lambda C^{\prime \prime}\left(F_{x}^{-1}(z)\right)-G^{\prime}(z) u^{\prime \prime}\left(w_{0}-Q\right)} .
$$

In order to satisfy the first constraint in Model 5, we transform $k(z)$ into

$$
K(z)=\max \left\{0, \min \left\{J(z), F_{x}^{-1}(z)\right\}\right\}, \quad 0 \leq z \leq 1 .
$$

Noting $u^{\prime}(\cdot) \geq 0, u^{\prime \prime}(\cdot) \leq 0$, and Assumption 4, we can find that $J(z)$ is nondecreasing on $\left[0, z_{0}\right]$ which satisfies the proposition of the quantile function. Hence, $K(z)$ satisfies the second constraint in Model 5 on $\left[0, z_{0}\right]$. However, it is regretful that $J(z)$ is not nondecreasing on $\left(z_{0}, 1\right]$ and we cannot make sure that $K(z)$ nondecreasing on $\left(z_{0}, 1\right]$. So, we hope to achieve a new solution suitable for all constraints in Model 5 from $K(z)$. We only pay attention to the case of $J(z)$ in $\left(z_{0}, 1\right]$. Since, in $\left(z_{0}, 1\right], J(z)$ is not always decreasing or increasing, we divide $\left(z_{0}, 1\right]$ by monotonicity. If $\left(z_{0}, 1\right]$ has $N$ increasing ranges and $M$ decreasing ranges, we denote increasing range by $\left(d_{i}, e_{i}\right](i=0,1, \ldots, N-1)$ and a decreasing range by $\left(e_{j}, d_{j+1}\right](j=0, \ldots, M-1)$ (see Figure 2).

In the first case in Figure 2, we can safely claim that $J(z)$ is nondecreasing on $\left[0, z_{0}\right]$ and $\left[z_{0}, e_{0}\right]\left(\left[z_{0}, e_{0}\right]\right.$ is equivalent to $\left.\left[d_{0}, e_{0}\right]\right)$. So, we can look at $\left[0, z_{0}\right]$ and $\left[z_{0}, e_{0}\right]$ as one whole; namely, we can deal with $J(z)$ on $\left[0, e_{0}\right]$ similar to that on $\left[0, z_{0}\right]$. At this time,we only need to discuss the rest part, $\left[e_{0}, 1\right] . J(z)$ first decreases on $\left[e_{0}, d_{1}\right]$ and then increases on $\left[d_{1}, d_{2}\right]$, which is the same as the second case in Figure 2. $J(z)$ first decreases and then increases in $\left(e_{0}, 1\right]$ and is similar to the second case in Figure 2.

We denote the intersection point of $F_{x}^{-1}(z)$ and $J(z)$ in $\left(e_{0}, d_{1}\right]$ by $s_{1}$. And write the intersection point of $k(z)$ and $J(z)$ on $\left[s_{1}, d_{1}\right]$ as $s_{2}$. Now, we discuss each possible case (see Figure 3).

Case 1. There exist $s_{1}$ and $s_{2}$ in $\left(e_{0}, d_{1}\right]$. At this time, we denote the intersection point of $k(z)$ and $F_{x}^{-1}(z)$ by $m_{0}$. Denote the intersection point of the horizontal line through $m_{0}$ and $J(z)$ on $\left[e_{0}, d_{1}\right]$ by $l_{0}$ and on $\left[d_{1}, e_{1}\right]$ by $n_{0}$.

Case 2. There exists $s_{1}$ and does not exist $s_{2}$ in $\left(e_{0}, d_{1}\right]$. In this case, we denote the intersection point of $k(z)$ (here $k(z)$ is equivalent to $\left.F_{x}^{-1}(z)\right)$ and the horizontal line through $d_{1}$ by $m_{0}$. And, set $l_{0}=n_{0}=d_{1}$.

Case 3. Consider $F_{x}^{-1}(z) \geq J(z)$ and there does not exist an intersection of $J(z)$ and $k(z)$ on $\left[z_{0}, d_{1}\right]$. In other words, there does not exist $s_{1}$. But we can look at $e_{0}$ as $s_{1}$, then this case is similar to the first case. We denote the intersection of $J(z)$ and $k(z)$ on $\left[z_{0}, d_{1}\right]$ by $s_{2}$. And, we denote the intersection point of $k(z)$ and the horizontal line through $s_{2}$ by $m_{0}$. It is valuably noticed that $k(z)$ is $J(z)$ at this time. In fact, $m_{0}$ is the intersection point of $J(z)$ and the horizontal line through $s_{2}$. Besides, denote the intersection point of the horizontal line through $s_{2}$ and $J(z)$ on $\left[d_{1}, e_{1}\right]$ by $n_{0}$ and $l_{0}=s_{2}$.

Case 4. Consider $F_{x}^{-1}\left(z_{0}\right) \geq J\left(z_{0}\right)$ and there does not exist an intersection of $J(z)$ and $k(z)$ on $\left[z_{0}, d_{1}\right]$. At this time, 

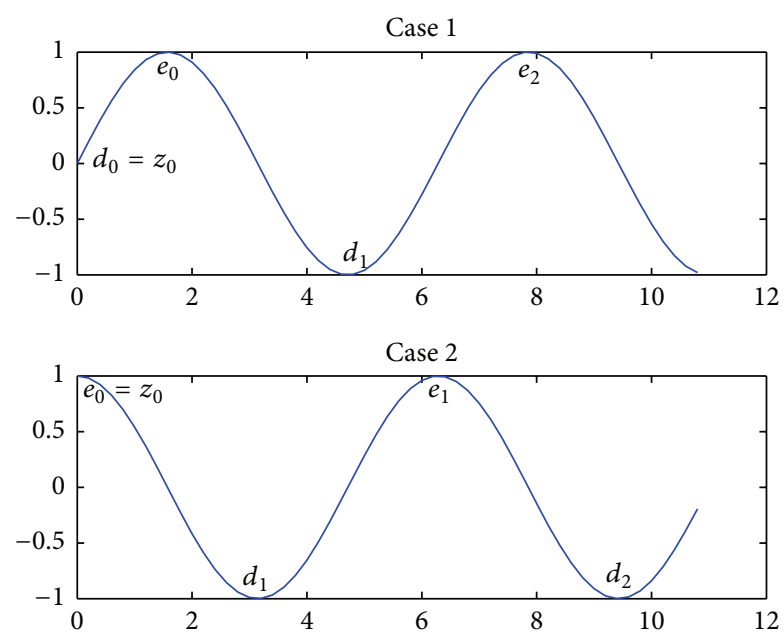

FIGURE 2

we denote the intersection point of $k(z)$ which is $J(z)$ in this case and the horizontal line through $d_{1}$ by $m_{0}$. And, let $l_{0}=n_{0}=d_{1}$.

Case 5. Consider $F_{x}^{-1}\left(d_{1}\right)<J\left(d_{1}\right)$. We denote the intersection of $F_{x}^{-1}(z)$ and the vertical line through $d_{1}$ by $m_{0}$. And, let $n_{0}=l_{0}=m_{0}$.

Let

$$
\begin{aligned}
K_{m_{0}}(z)=K(z) I_{0 \leq z \leq m_{0}}+K\left(m_{0}\right) I_{n_{0}>z>m_{0}}, & \\
& 0<z<n_{0} .
\end{aligned}
$$

Then, we can achieve an important lemma as follows.
Lemma 7. For the feasible solution $k(z)$ of Model $5, K_{m_{0}}(z)$ satisfies the following:

(i) $U_{\lambda}(k(z), z) \leq U_{\lambda}\left(K_{m_{0}}(z), z\right), 0<z<n_{0}$.

(ii) The equality holds if and only if $k(z)=K_{m_{0}}(z), 0<z<$ $n_{0}$.

The proof is seen in Appendix B.

3.2. The Global Optimal Insurance Design. For the range $\left[n_{0}, e_{2}\right]$, we can similary discuss to the range $\left[0, e_{1}\right]$. In more detail, we take $\left[n_{0}, e_{1}\right],\left[e_{1}, d_{2}\right]$, and $\left[d_{2}, e_{3}\right]$ as $\left[0, e_{0}\right],\left[e_{0}, d_{1}\right]$, and $\left[d_{1}, e_{2}\right]$, respectively. We can obtain $K_{m_{1}}(z)$ on $\left[n_{0}, n_{1}\right]$ similar to $K_{m_{0}}(Z)$ on $\left[0, n_{0}\right]$. Generally, we can achieve $K_{m_{i}}(z)$ on $\left[n_{i-1}, n_{i}\right]$ similar to $K_{m_{0}}(Z)$ on $\left[0, n_{0}\right](i=1,2, \ldots, N-$ 1 or $N-2)$.

Because we note that $k(z)$ must be the left-continuous, we are particularly concerned with whether the value of $K_{m_{1}}(z)$ equals $K_{m_{0}}(z)$ which directly decides whether $k(z)$ is the leftcontinuous. That is, does the following equality

$$
K_{m_{1}}\left(n_{0}\right)=K_{m_{0}}\left(n_{0}\right)
$$

hold?

In the first four cases, we can find $F_{x}^{-1}\left(n_{0}\right) \geq J\left(n_{0}\right)$. So, $K\left(n_{0}\right)=J\left(n_{0}\right)$ and $K_{m_{0}}\left(n_{0}\right)=J\left(n_{0}\right)=K\left(n_{0}\right)=K_{m_{1}}\left(n_{0}\right)$. In the last case, we have $F_{x}^{-1}\left(n_{0}\right) \leq J\left(n_{0}\right)$. So, $K\left(n_{0}\right)=F_{x}^{-1}\left(n_{0}\right)$ and $K_{m_{0}}\left(n_{0}\right)=F_{x}^{-1}\left(n_{0}\right)=K\left(n_{0}\right)=K_{m_{1}}\left(n_{0}\right)$.

Hence, the function $K_{m_{0}}(z) I_{0 \leq z \leq n_{0}}+K_{m_{1}}(z) I_{n_{0}<z \leq n_{1}}$ on $\left[0, n_{1}\right]$ is nondecreasing and left-continuous. Generally, the range $\left[n_{i-1}, n_{i}\right]$ is researched similarly. Ultimately, noticing (35), we can indicate the nondecreasing and left-continuous function $\bar{K}(z)$ on $[0,1]$ as follows:

$$
\bar{K}(z)= \begin{cases}K_{m_{0}}(z) I_{0 \leq z \leq n_{0}}+K_{m_{1}}(z) I_{n_{0}<z \leq n_{1}}+\cdots+K_{m_{N-1}}(z) I_{n_{N-2}<z \leq 1} & \text { if } M=N \\ K_{m_{0}}(z) I_{0 \leq z \leq n_{0}}+\cdots+K_{m_{N-2}}(z) I_{n_{N-3}<z \leq n_{N-2}}+K(z) I_{n_{N-2}<z \leq 1} & \text { if } M<N .\end{cases}
$$

Now, we can attain the following conclusion.

Theorem 8. For and feasible solution $k(z)$ of Model $5, \bar{K}(z)$ satisfies the following:

(i) $U_{\lambda}(k(z), z) \leq U_{\lambda}(\bar{K}(z), z), 0<z<1$.

(ii) The equality holds if and only if $k(z)=\bar{K}(z), 0<z<1$.

The proof is seen in Appendix C.

From Theorem 8, we can reduce Model 5 to the auxiliary problem with Lagrange dual method.

Model 6. Consider

$$
\begin{aligned}
\operatorname{Max}_{k(z)} U_{\lambda}(k(z), z)= & \operatorname{int}_{0}^{1} u\left(w_{0}-Q-k(z)\right) G^{\prime}(z) \\
& +\lambda k(z)
\end{aligned}
$$

$$
\begin{aligned}
& -\lambda C\left(F_{x}^{-1}(z)-k(z)\right) d z \\
& +\lambda(Q-E x),
\end{aligned}
$$

where $k(z)$ is satisfied to (36).

Now, we desire to solve the original optimal problem through $U_{\lambda}(\bar{K}(z), z)$. We can attain the key proposition as follows.

Proposition 9. The optimal solution of Model 1 is as follows:

$$
\begin{aligned}
& I^{*}(x)=x-K\left(n_{i}\right) I_{n_{i+1}>z>n_{i}}, \\
& F_{x}^{-1}\left(n_{i+1}\right)>x>F_{x}^{-1}\left(n_{i}\right),
\end{aligned}
$$

where

$$
K\left(n_{i}\right) I_{n_{i+1}>z>n_{i}}=K_{n_{i}}(z)=K_{m_{i+1}}(z) .
$$



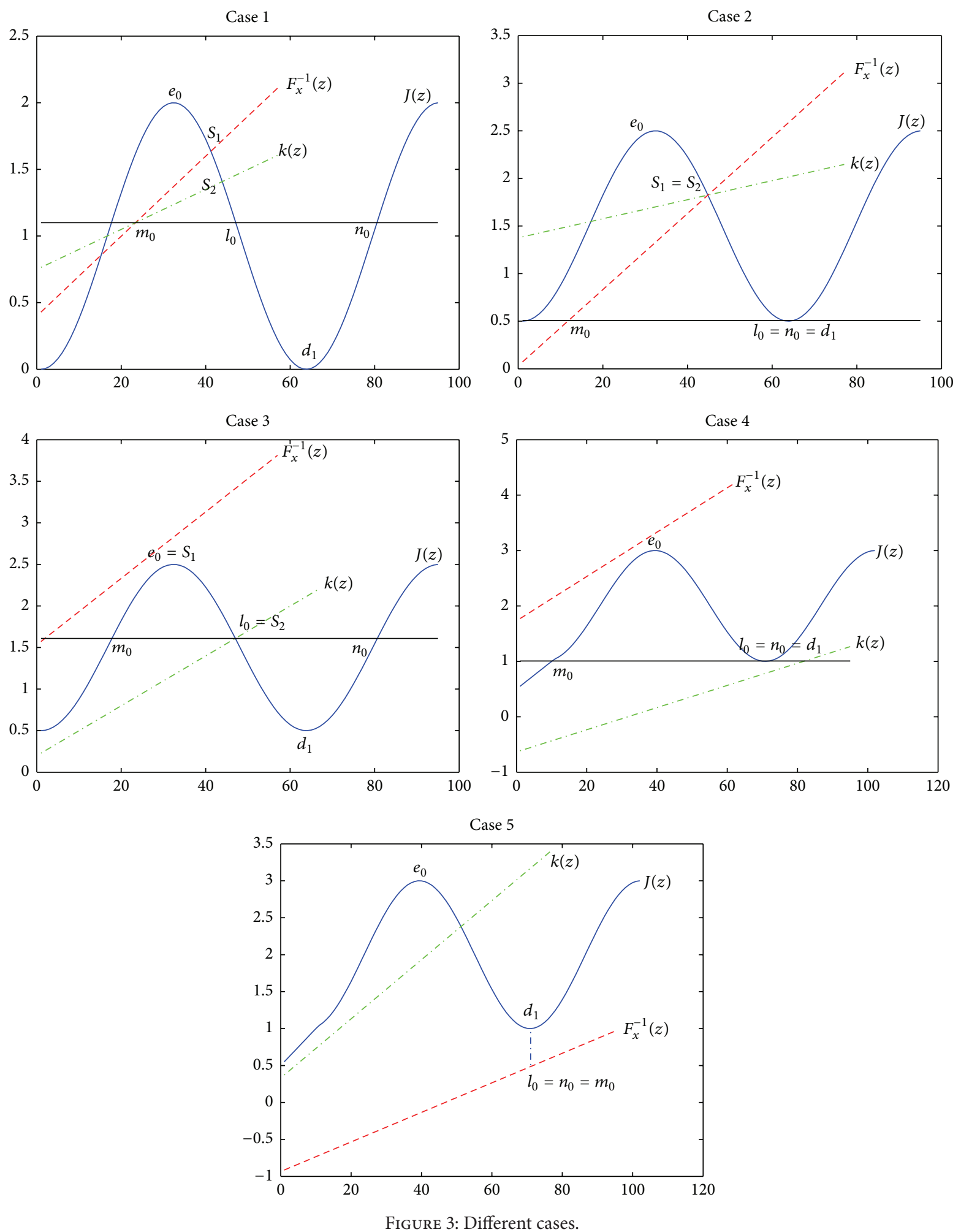

The proof is seen in Appendix D.

Remark 10. Comparing the conclusion with the result in the substance of Bernard and Zhou's paper, we cannot help asking why does our conclusion seem simpler than Zhou and
Bernard's one, while our fixed cost of handling an indemnity is more general than Bernard's research? It is because we subtly make $J(z)$ become segments, $\left[n_{i}, n_{i+1}\right]$, before solving this optimal problem which delicately simplifies the form of solution. 


\section{Numerical Analysis}

In this section, we provide an insight into the optimal result with a numerical simulation. We suppose the loss $x$ satisfies truncated Pareto distribution on $\left[0, w_{0}\right]$. That is, the density function is

$$
f(x)= \begin{cases}\frac{1}{1-2^{-\alpha}} \frac{\alpha}{w_{0}}\left(\frac{w_{0}}{2 w_{0}-x}\right)^{\alpha+1} & 0 \leq x \leq w_{0} \\ 0 & \text { others. }\end{cases}
$$

Accordingly, its distribution function is

$$
\begin{aligned}
F(x) & =\int_{0}^{x} f(t) d t \\
& =\int_{0}^{x} \frac{1}{1-2^{-\alpha}} \frac{\alpha}{w_{0}}\left(\frac{w_{0}}{2 w_{0}-t}\right)^{\alpha+1} d t \\
& =\left.\frac{1}{1-2^{-\alpha}} w_{0}^{\alpha}\left(2 w_{0}-t\right)^{-\alpha}\right|_{0} ^{x} \\
& =\frac{1}{1-2^{-\alpha}} w_{0}^{\alpha}\left[\left(2 w_{0}-x\right)^{-\alpha}-\left(2 w_{0}\right)^{-\alpha}\right] .
\end{aligned}
$$

$$
\begin{aligned}
G^{\prime}(z) & =\frac{\gamma z^{\gamma-1}\left(z^{\gamma}+(1-z)^{\gamma}\right)^{1 / \gamma}-z^{\gamma}(1 / \gamma)\left(z^{\gamma}+(1-z)^{\gamma}\right)^{1 / \gamma-1}\left(\gamma z^{\gamma-1}-\gamma(1-z)^{\gamma-1}\right)}{\left(z^{\gamma}+(1-z)^{\gamma}\right)^{2 / \gamma}}, \\
\gamma & \approx 0.61 .
\end{aligned}
$$

In the body of this section, we mainly focus on the optimal solutions' sensitivity to the fixed cost function of handling an indemnity and the initial wealth of the insured. Here, we choose the utility function of the insured $u(w)=\log w$.

Firstly, we consider that different parameters in the fixed cost function of an indemnity impact an optimal insurance contract. We fix the initial wealth of the insured $w_{0}=7$, the premium $Q=0.3 w_{0}=2.1$, and $b=1$. By setting $a=$ $0.1,0.5,2.0,15.0$ (See Figure 4), we display that the optimal indemnity depends on the values of $a$ since the change of $a$ effects curvature of $J(z)$. Particularly, as for large losses and small losses, the larger $a$ becomes, the higher the limit of the indemnity becomes and the smaller the deductible becomes. Meanwhile, as for medium losses, when the loss is larger than the fixed threshold value (here the fixed threshold value is about 1.0), the insurer will fully pay for the losses. When the loss is smaller than this fixed threshold value, there is partial reimbursement. With increasing the value of $a$, the proportion of the partial payment becomes higher. Until $a=15.0$, the proportion of the partial indemnity almost reaches 1; that is, the partial reimbursement becomes the full payment. Moreover, when $a=15.0$, the limit of the indemnity nearly runs up to 3.0 and the deductible almost disappears. In a word, the raise of the parameter $a$ makes the insurance contract more beneficial for the insured. In particular, when $a=15.0$, the insured can get
As for the insured's utility function, we discuss two different kinds of utility functions. They are separately

$$
\begin{aligned}
& u(w)=\frac{1}{1-\beta} w^{1-\beta} \\
& u(w)=\log w
\end{aligned}
$$

We let the fixed cost function of handling an indemnity be

$$
C(I)=\frac{a}{2} I^{2}+b I+c, \quad a, b \geq 0
$$

The probability weighting function (see [10]) is

$$
G(z)=\frac{z^{\gamma}}{\left(z^{\gamma}+(1-z)^{\gamma}\right)^{1 / \gamma}}, \quad \gamma \approx 0.61 .
$$

Then,

full reimbursement under the limit of the indemnity without the deductible.

Sequently, we also carry out the sensitivity of an optimal solution to $b$. Similar to the above, we fix the initial wealth of the insured $w_{0}=7$, the premium $Q=0.3 w_{0}=2.1$, and $a=0.5$ first. Then, let $b=0.1,0.3,1.0,2.0$ (see Figure 5). Figure 5 describes that $b$ dominates the values of $J(z)$ rather than the shape of $J(z)$ which leads to the change of optimal solutions. Particularly, for large losses, with increasing the value of $b$, the limit of recovery rises. This effect of $b$ is similar to $a$, but the impact of $b$ is not more obvious than a. Simultaneously, as for small losses, the raise of $b$ also decreases the deductible. Of course, we also notice medium losses. With our best endeavors, it is not difficult to show that when the loss is larger than a threshold value, the insured will get full reimbursement. When the loss is smaller than this threshold value, there is a partial payment. It is necessary to make a special note that this threshold is distinguished from the threshold value in the case of the parameter $a$. This threshold increases with the value of $b$ raising. At the same time, the proportion of the partial indemnity does not depend on the parameter $b$, which is different from the case of the parameter $a$. Until $b=2.0$, the deductible almost reaches 0 and the limit of the indemnity nearly runs up to the maximal value that is about 3.0. In short, the raise of $b$ is more advantageous for the insured. In particular, when $b=2.0$, 

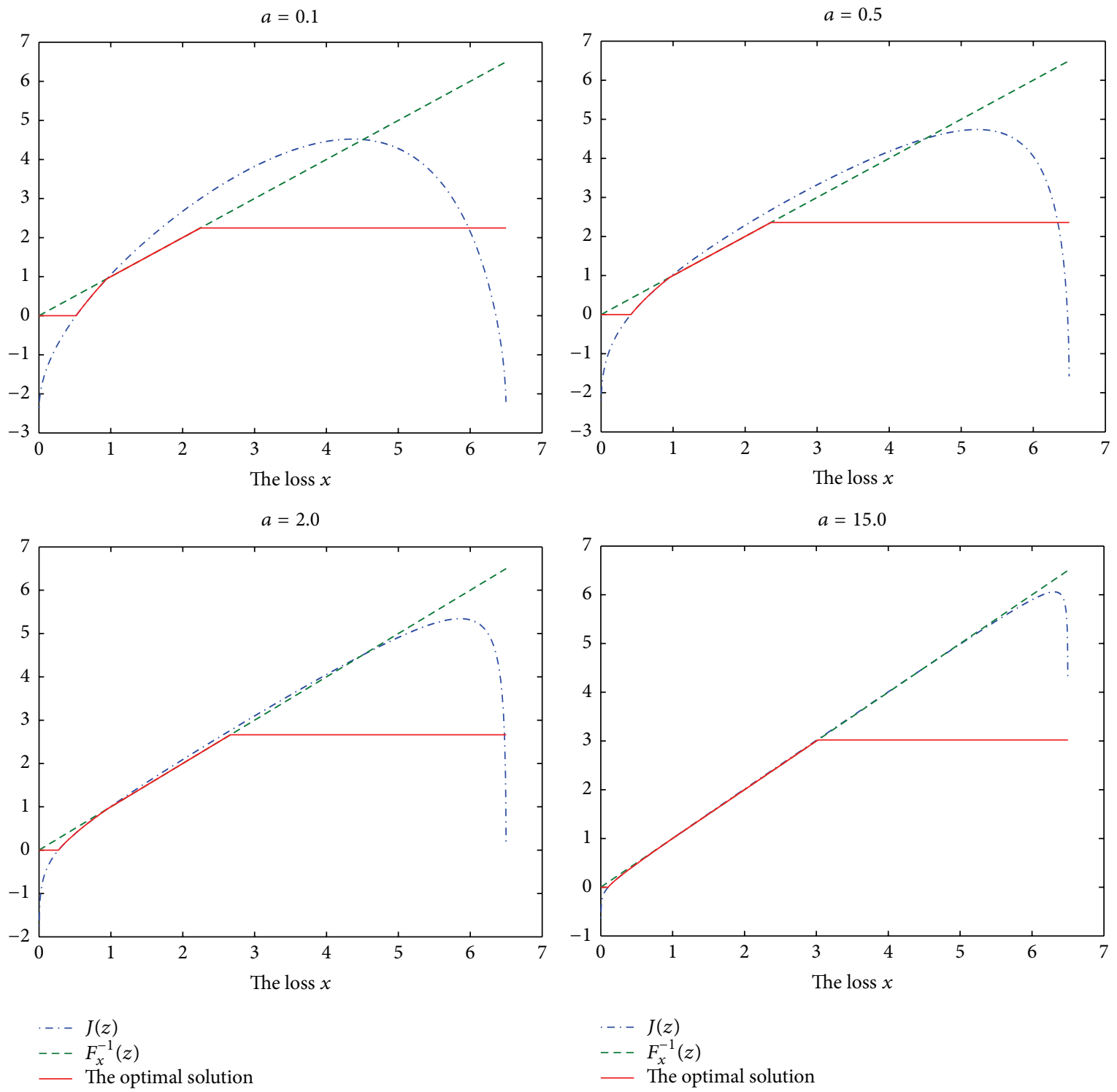

Figure 4: Different parameters of the cost $a$.

the insured can get full reimbursement under the limit of the indemnity without the deductible.

Now, we are interested in the influence of the insured's initial wealth on the optimal solution. Similar to the above parameters, we fix the premium $Q=0.3 w_{0}, a=0.5$, and $b=1.0$ and set $w_{0}=4.5,5.2,8.0,20$. It is a surprise to find the different relationship between $J(z)$ and $F_{x}^{-1}(z)$ (see Figure 6) with different values of the initial wealth of insured $w_{0}$. It is not difficult to obtain the optimal solutions, through the above discussion of five different cases in Section 3 (see Figure 3). The computation of the optimal solution identifies the independent threshold value which plays the pivotal role in relationship between $J(z)$ and $F_{x}^{-1}(z)$ and the choice of the optimal contract. This threshold is $w_{0}=5.2$ in Figure 6. In particular, when $w_{0}=5.2, F_{x}^{-1}(z)$ tangents to $J(z)$. When $w_{0}<5.2, J(z)$ and $F_{x}^{-1}(z)$ are disjoined. When $w_{0}>5.2$, $F_{x}^{-1}(z)$ intersects $J(z)$. The various relationships between $J(x)$ and $F_{x}^{-1}(z)$ makes us attain the different optimal solutions. Specifically, when $w_{0}=4.5,5.2$, three different segments constitute the optimal solution, which, respectively, represent the limit of indemnity, deductible, and the full payment for medium losses. It is valuably noticed that, comparing $w_{0}=$ 4.5 and $w_{0}=5.2$, the limit of recovery is higher in the case of $w_{0}=5.2$ than the one in the case of $w_{0}=4.5$. Another important point, as we think, is that all of the optimal solutions when $w_{0}<5.2$ are the same. The optimal solutions consist of four segments, when $w_{0}=7.0, w_{0}=8.0$, and $w_{0}=15.0$. These cases are more complicated than the cases of $w_{0}=4.5$ and $w_{0}=5.2$, since there are other threshold values between full and partial indemnity for medium losses. Meanwhile, we emphasize that, with increasing the value of $w_{0}$, the threshold value for medium losses becomes smaller and smaller. Further, this rise of the initial wealth of the insured $w_{0}$ makes the deductible becomes smaller. Until $w_{0}=20$, 

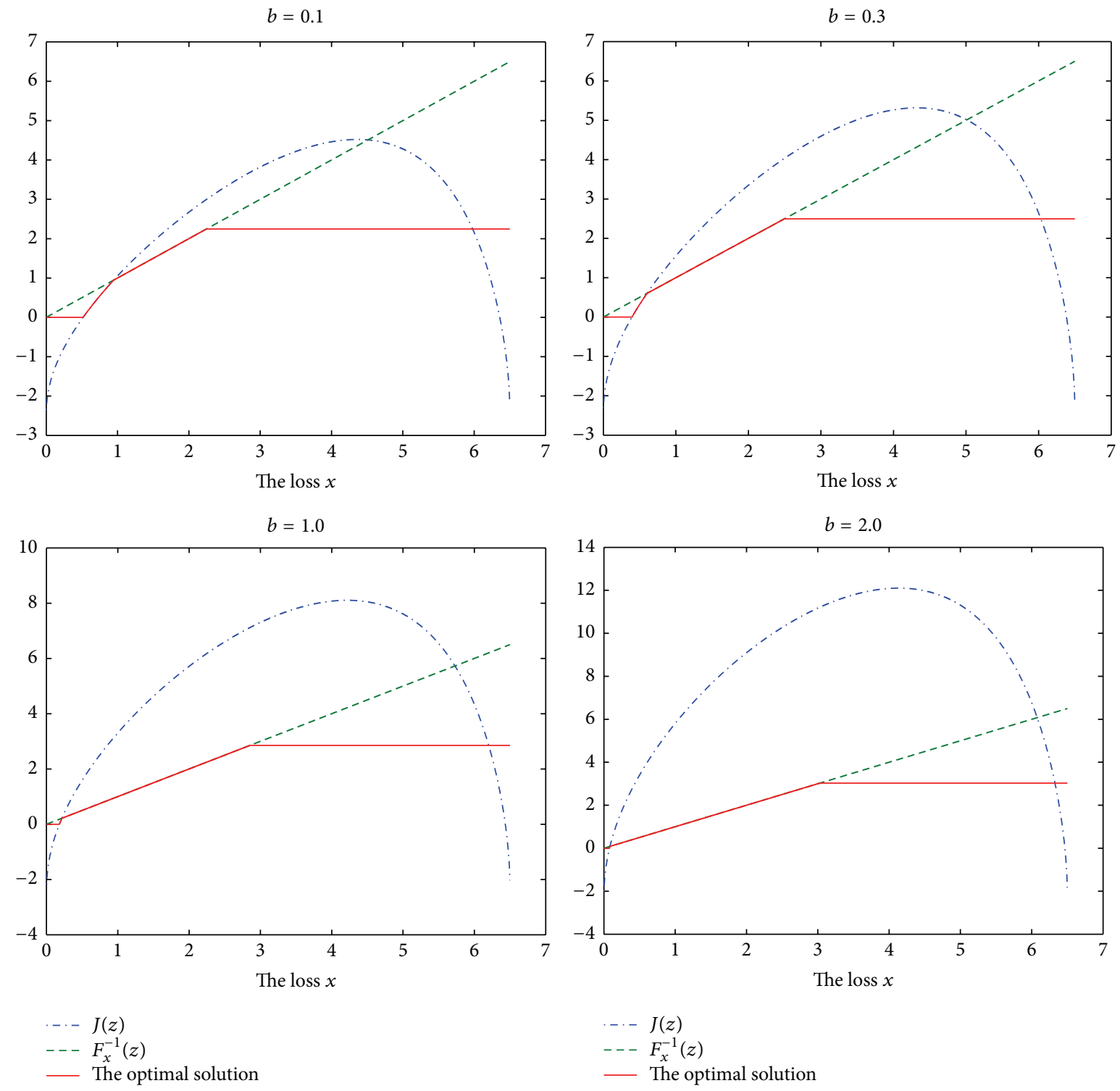

FIGURE 5: Different parameters of the cost $b$.

both the threshold for medium losses and the deductible scarcely exit. Simultaneously, the limit of the recovery nearly reaches the maximal value which is about 10.0. Namely, all of the losses under 10 can be fully reimbursed at this time.

After testing the sensitivity of the optimal insurance policy, we compare the different utility functions of the insured in terms of the influence of the optimal solution. We fix necessary parameters, the initial wealth of the insured $w_{0}=4.5$, the premium $Q=0.3 w_{0}=2.1, a=0.5$, and $b=1$. Figure 7 indicates that the various utility functions do not change the shapes of $J(z)$ expect for shifting up. This shift brings the optimal insurance contract some tiny distinctions. When

$$
u(w)=\frac{1}{1-\beta} w^{1-\beta}
$$

the numerical analysis reveals that the limit of the recovery is about 2.0 and the insurer must fully pay for losses under the limit of the indemnity. Meanwhile, when

$$
u(w)=\log w,
$$

there are a small deductible for small losses and a partial payment for medium losses.

\section{Conclusion}

In contrast with Bernard et al.s work, our main contributions are main two aspects. On the one hand, we generalize the fixed cost functions. Besides, through the mathematically sophisticated and complicated derivation of solution, we state the considerably surprising and subtle solution in explicit form for both the linear cost functions and the nonlinear cost functions. The shortcoming of prospect theory 
$w_{0}=4.5$
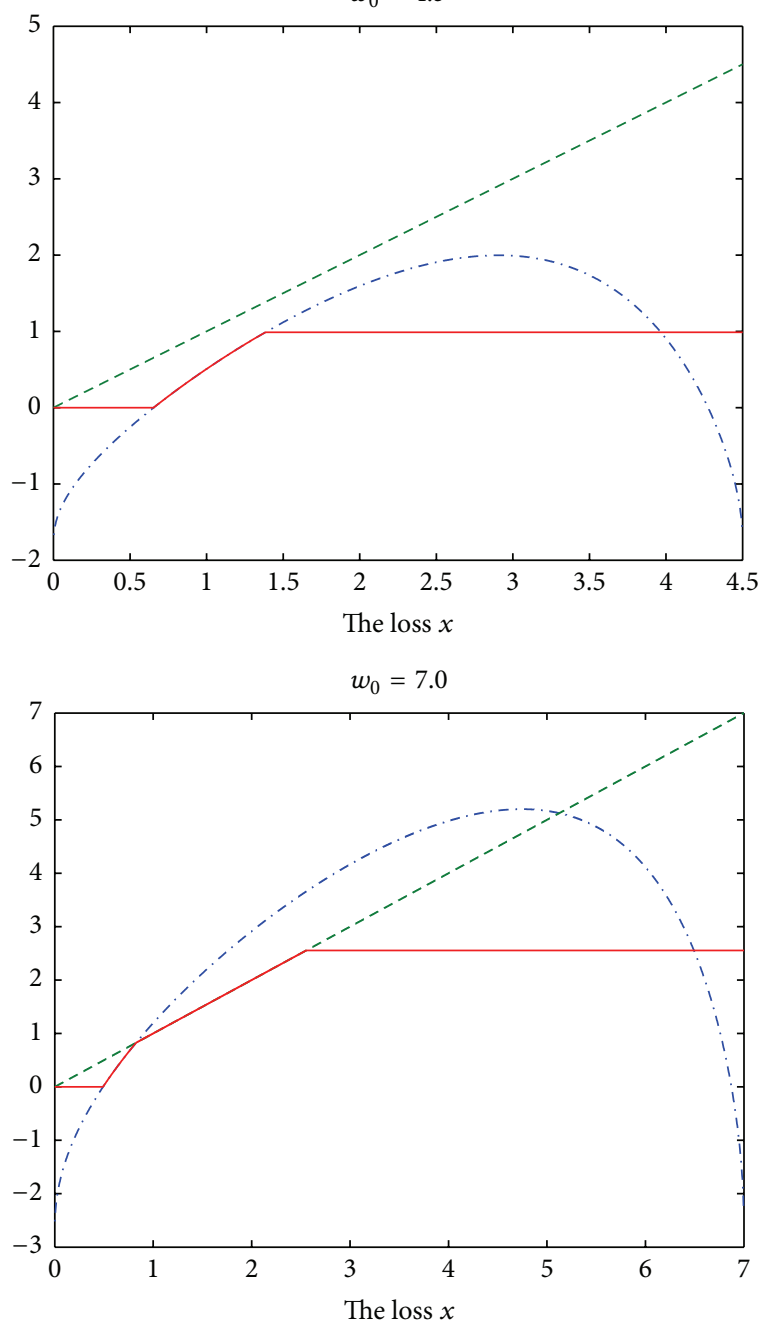

$w_{0}=15$

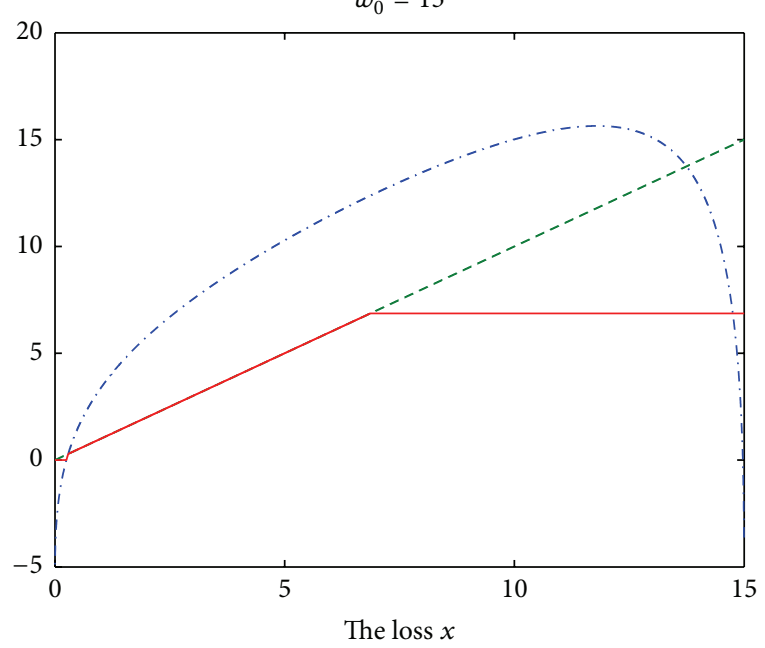

-. - J(z)

$--F_{x}^{-1}(z)$

- The optimal solution $w_{0}=5.2$

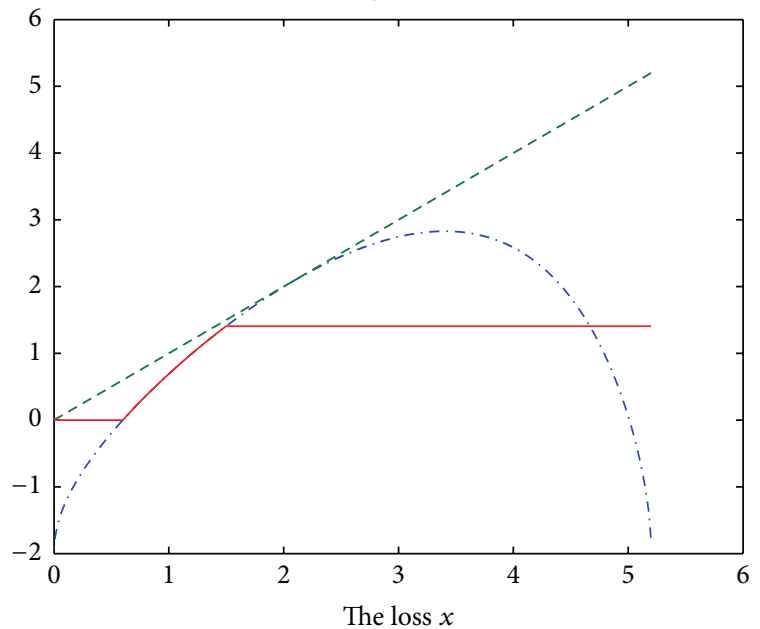

$w_{0}=8.0$

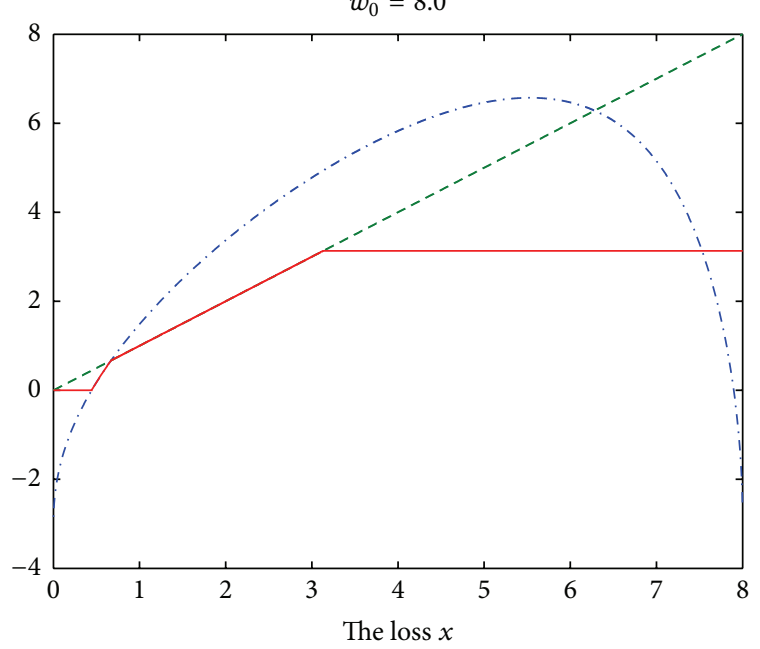

$w_{0}=20$

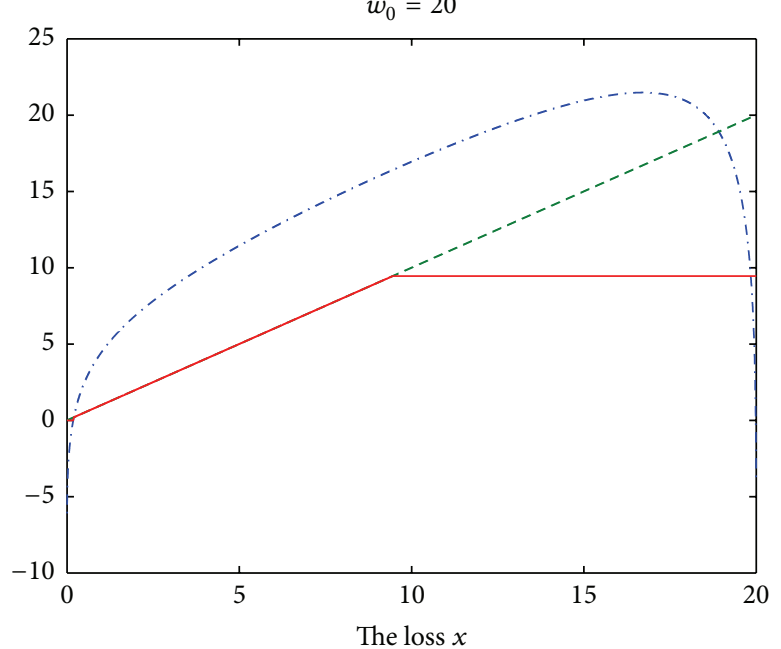

$-\cdot-J(z)$

$--F_{x}^{-1}(z)$

The optimal solution

Figure 6: Different initial wealth of the insured. 

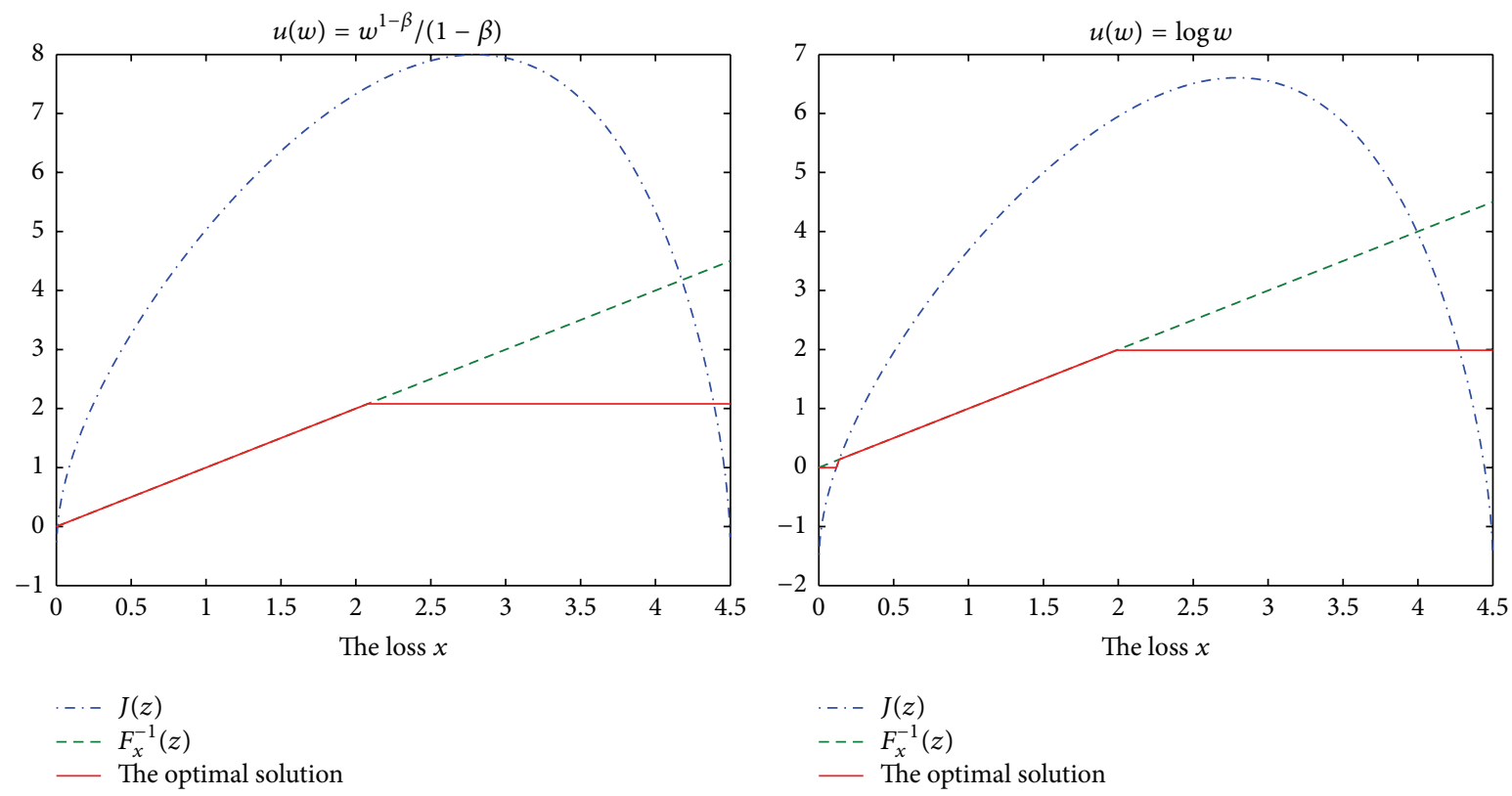

FIgURE 7: Different utility functions of the insured.

is that a conventional well-posed problem becomes an illposed problem with prospect theory. The nonexistence of an explicit solution impedes the progress in the application and impact of prospect theory. Hence, the generally novel results are meaningful and significant. On the other hand, The adverse selection and moral hazard are considered by us while Bernard et al. do not involve them. We use bonus-malus system and the empirical test to decide the premium $Q$. Based on the fixed $Q$, we further explore the optimal insurance design under Rand-Dependent Expected Utility. Because we focus on the optimal insurance contract for the general cost functions, we only provide a brief thought of how to determine the premium $Q$ and not carefully study it. However, this significant idea not only makes close relationship between these critical issues and our research but also offers the basic framework for the further research.

Compared with the Ben Dhiab's research, our novelty is researched from the different perspective. Particularly, he researches the optimal insurance contract from the insured's (or agent's) point of view, so he only needs to maximize the RDEU of the insured without considering the utility function of an insurer and relative restrictive conditions. However, we study the optimal insurance policy from an insurer's point of view. Furthermore, I reveal the accurately quantified relationship between the indemnity function $I(x)$ and the random loss $x$ while he does not present the quantified relationship between the indemnity function $I(x)$ and the random loss $x$ in the cases of partial insurance and overinsurance. On the technological detail, we employ the $S$ shape probability weighting function and suppose that the loss $x$ is a random variable on $\left[0, w_{0}\right]$, whereas Ben Dhiab only assumes that the probability weighting function is always concave or convex and the loss $x$ with probability $p$ and no-loss with probability $1-p$. Sufficient evidences state that our assumptions are more practical than his.

In the numerical aspect, we tested the sensitivity of an optimal insurance contract for the fixed cost function of handling the indemnity, the initial wealth of the insured, and two different utility functions of the insured in the numerical means. For the parameter $a$, raising the value of $a$ makes the insurance policy more beneficial for the insured, because the raising the parameter $a$ can increase the limit of recovery and the proportion of indemnity for medium losses. Meanwhile, raising $a$ can decrease the deductible. Similar to the parameter $a$, increasing the parameter $b$ also brings the insured more profit. It is the main reason that increasing parameter $b$ can increase the limit of reimbursement and reduce the deductible while the larger parameter $b$ makes the threshold value between full and partial payments become smaller and the partial reimbursement be almost replaced by full indemnity.

\section{Further Research}

Although we have achieved notable and novel findings, this research is not perfect. For example, we only involve two kinds of utility functions of the insured and do not discuss others. As for different utility functions, we believe the sensitivity of optimal insurance design for the fixed cost of handling the indemnity and the initial wealth of the insured is different. But, we only emphasize the sensitivity of utility function $u(w)=\log (w)$. Besides, the alterable parameters in our problem are far more than the illustrated parameters $a$, $b$, and $w_{0}$ by us. Other parameters' variety, we believe, will impact the optimal solution.

In the optimal insurance contract, there are some important game relationships, which are the relationship between 
principal-agent, agent-agent, and principal-principal. In the latter research, we will pay attention to these game relationships. Particularly, based on Sappington's frameworks, we will combine the monitoring and competition with the optimal insurance contract under Rank-Dependent Expected Utility. Besides, considering the critical issues, adverse selection, and moral hazard in the optimal insurance, we will apply bonusmalus system and empirical tests to decide the premium $Q$ and further research the dynamic optimal insurance design relying on the fixed the premium $Q$.

\section{Appendices}

\section{A. The Proof of Lemma 5}

Proof. Suppose the probability density function of $A-g(x)$ is $H(\cdot)$. Noticing that

$$
\begin{aligned}
f(t) & =f(g(x)=t)=f(A-g(x)=A-t) \\
& =H(A-t),
\end{aligned}
$$

we have sound reason to state that

$$
\int_{-\infty}^{A-t} H(y) d y=1-\int_{-\infty}^{t} f(y) d y .
$$

With the definition of the cumulative distribution function, it is easy to see that

$$
\begin{aligned}
F_{g(x)}(t) & =\int_{-\infty}^{t} f(x) d x, \\
F_{a-g(x)}(A-t) & =\int_{-\infty}^{A-t} H(x) d x .
\end{aligned}
$$

Let

$$
z=F_{a-g(x)}(A-t)=\int_{-\infty}^{A-t} H(x) d x .
$$

With (A.2), we have

$$
1-z=F_{g(x)}(t)=\int_{-\infty}^{t} f(x) d x .
$$

Hence,

$$
\begin{aligned}
A-t & =F_{A-g(x)}^{-1}(z), \\
t & =F_{g(x)}^{-1}(1-z) .
\end{aligned}
$$

So,

$$
F_{A-g(x)}^{-1}(z)=A-F_{g(x)}^{-1}(1-z)
$$

\section{B. The Proof of Lemma 7}

Proof. To simply write this proof, we set

$$
\begin{aligned}
h(k(z), z)= & u\left(w_{0}-Q-k(z)\right) G^{\prime}(z)+\lambda k(z) \\
& -\lambda C\left(F_{x}^{-1}(z)-k(z)\right) .
\end{aligned}
$$

Then,

$$
\begin{aligned}
& U_{\lambda}(k(z), z)=\int_{0}^{n_{0}} u\left(w_{0}-Q-k(z)\right) G^{\prime}(z) \\
& +\lambda k(z)-\lambda C\left(F_{x}^{-1}(z)-k(z)\right) d z+\lambda(Q \\
& \quad-E x)=\int_{0}^{n_{0}} h(k(z), z) d z+\lambda(Q-E x) \\
& \quad=\int_{0}^{m_{0}} h(k(z), z) d z+\int_{m_{0}}^{l_{0}} h(k(z), z) d z \\
& +\int_{l_{0}}^{n_{0}} h(z)(k(z), z) d z+\lambda(Q-E x)=I_{1}+I_{2} \\
& +I_{3}+\lambda(Q-E x) .
\end{aligned}
$$

It is easy to see that $K(z)$ is the unique maximal value of $h(k(z), z)$ on $\left[0, F_{x}^{-1}(z)\right]$ for each fixed $z \in\left(0, n_{0}\right)$. So, $h(k(z), z)(x)$ is strictly increasing on $[0, K(z)]$ and strictly decreasing on $\left[K(z), F_{x}^{-1}(z)\right]$.

We firstly discuss $I_{1}$. On $\left[0, m_{0}\right]$, if $k(z) \geq K(z)$, then $k(z) \in\left[K(z), F_{x}^{-1}(z)\right]$. Since $h(k(z), z)$ is strictly decreasing on $\left[K(z), F_{x}^{-1}(z)\right]$,

$$
h(k(z), z) \leq h(K(z), z)=h\left(K_{m_{0}}(z), z\right) ;
$$

if $k(z) \leq K(z)$, then $k(z) \in[0, K(z)]$. Since $h(k(z), z)$ is strictly increasing on $[0, K(z)]$,

$$
h(k(z), z) \leq h(K(z), z)=h\left(K_{m_{0}}(z), z\right) .
$$

Therefore, we have

$$
\begin{aligned}
I_{1} & =\int_{0}^{m_{0}} h(k(z), z) d z \leq \int_{0}^{m_{0}} h(K(z), z) d z \\
& =\int_{0}^{m_{0}} h\left(K_{m_{0}}(z), z\right) d z .
\end{aligned}
$$

In the next discussion, we focus on $I_{2}$. On $\left[m_{0}, l_{0}\right]$,

$$
k(z) \leq K_{m_{0}}(z)=K\left(m_{0}\right) \leq K(z) .
$$

Consider $k(z) \in[0, K(Z)]$ and $K_{m_{0}}(z) \in[0, K(Z)]$; hence, $h(k(z), z) \leq h\left(K_{m_{0}}(z), z\right)$. That is,

$$
I_{2}=\int_{m_{0}}^{l_{0}} h(k(z), z) d z \leq \int_{m_{0}}^{l_{0}} h\left(K_{m_{0}}(z), z\right) d z .
$$

As for $I_{2}$, noticing under Cases 2, 4, and $5 n_{0}=l_{0}$, we have

$$
\begin{aligned}
I_{3} & =\int_{l_{0}}^{n_{0}} h(z)(k(z), z) d z \\
& =\int_{l_{0}}^{n_{0}} h(z)\left(K_{m_{0}}(z), z\right) d z=0 .
\end{aligned}
$$

Under Cases 1 and 3, we have sound reason to state that

$$
K(z) \leq K_{m_{0}}(z)=K\left(m_{0}\right) \leq k(z)
$$

on $\left[l_{0}, n_{0}\right]$. 
Since $h(k(z), z)$ is strictly decreasing on $\left[K(z), F_{x}^{-1}(z)\right]$,

$$
h(k(z), z) \leq h(K(z), z)=h\left(K_{m_{0}}(z), z\right) .
$$

So,

$$
I_{3}=\int_{l_{0}}^{n_{0}} h(k(z), z) d z \leq \int_{l_{0}}^{n_{0}} h\left(K_{m_{0}}(z), z\right) d z .
$$

Now, we come to (B.2); it is easy to see that

$$
\begin{aligned}
U_{\lambda}(k(z), z)= & I_{1}+I_{2}+I_{3}+\lambda(Q-E x) \\
\leq & \int_{0}^{m_{0}} h\left(K_{m_{0}}(z), z\right) d z \\
& +\int_{m_{0}}^{l_{0}} h\left(K_{m_{0}}(z), z\right) d z \\
& +\int_{l_{0}}^{n_{0}} h\left(K_{m_{0}}(z), z\right) d z \\
& +\lambda(Q-E x) \\
= & \int_{0}^{n_{0}} h\left(K_{m_{0}}(z), z\right) d z+\lambda(Q-E x) \\
= & U_{\lambda}\left(K_{m_{0}}(z), z\right)
\end{aligned}
$$

and the equality holds if and only if $k(z)=K_{m_{0}}(z), 0<z<$ $n_{0}$.

\section{The Proof of Theorem 8}

Proof. Recalling Lemma 7, we can achieve a more general result

For feasible solution $k(z)$ of Model $5, K_{m_{i}}(z)$ satisfies the following:

(i) $U_{\lambda}(k(z), z) \leq U_{\lambda}\left(K_{m_{i}}(z), z\right), n_{i-1}<z<n_{i}$.

(ii) The equality holds if and only if $k(z)=K_{m_{0}}(z), n_{i-1}<$ $z<n_{i}$.

Therefore, when $N=M$, we have

$$
\begin{aligned}
& U_{\lambda}(k(z), z)=\int_{0}^{1} h(k(z), z) d z+\lambda(Q-E x) \\
& =\int_{0}^{n_{0}} h(k(z), z) d z+\int_{n_{0}}^{n_{1}} h(k(z), z) d z+\cdots \\
& \quad+\int_{n_{N-3}}^{n_{N-2}} h(z)(k(z), z) d z \\
& \quad+\int_{n_{N-2}}^{1} h(z)(k(z), z) d z+\lambda(Q-E x) \\
& \leq \int_{0}^{n_{0}} h\left(K_{m_{0}}(z) I_{0 \leq z \leq n_{0}}, z\right) d z \\
& \quad+\int_{n_{0}}^{n_{1}} h\left(K_{m_{1}}(z) I_{n_{0}<z \leq n_{1}}, z\right) d z+\cdots \\
& \quad+\int_{n_{N-3}}^{n_{N-2}} h(z)\left(K_{m_{N-2}}(z) I_{n_{N-3}<z \leq n_{N-2}}, z\right) d z
\end{aligned}
$$

$$
\begin{aligned}
& +\int_{n_{N-2}}^{1} h(z)\left(K_{m_{N-1}}(z) I_{n_{N-2}<z \leq 1}, z\right) d z \\
& +\lambda(Q-E x) \\
= & \int_{0}^{1} h(\bar{K}(z), z) d z+\lambda(Q-E x) \\
= & U_{\lambda}(\bar{K}(z), z)
\end{aligned}
$$

and the equality holds if and only if $k(z)=\bar{K}(z), 0<z<1$.

When $N \neq M$, we slip the detailed proof, since the result is derived in a similar means to the above case of $N=M$.

\section{The Proof of Proposition 9}

Proof. Due to $\bar{K}(z)$ being piecewise function, we take $K_{m_{i+1}}(z)$ on $\left[n_{i}, n_{i+1}\right]$ into account without loss of generality. Consider

$$
\begin{aligned}
& U_{\lambda}\left(K_{m_{i+1}}(z), z\right) \\
& =\int_{n_{i}}^{n_{i+1}} u\left(w_{0}-Q-K_{m_{i+1}}(z)\right) G^{\prime}(z) \\
& +\lambda\left(K_{m_{i+1}}(z)-C\left(F_{x}^{-1}(z)-K_{m_{i+1}}(z)\right)\right) d z \\
& +\lambda(Q-E x) \\
& =\int_{n_{i}}^{m_{i+1}} u\left(w_{0}-Q-K_{m_{i+1}}(z)\right) G^{\prime}(z) \\
& +\lambda\left(K_{m_{i+1}}(z)-C\left(F_{x}^{-1}(z)-K_{m_{i+1}}(z)\right)\right) d z \\
& +\int_{m_{i+1}}^{n_{i+1}} u\left(w_{0}-Q-K_{m_{i+1}}(z)\right) G^{\prime}(z) \\
& +\lambda\left(K_{m_{i+1}}(z)-C\left(F_{x}^{-1}(z)-K_{m_{i+1}}(z)\right)\right) d z \\
& +\lambda(Q-E x)=\int_{n_{i}}^{m_{i+1}} u\left(w_{0}-Q-K(z)\right) G^{\prime}(z) \\
& +\lambda\left(K(z)-C\left(F_{x}^{-1}(z)-K(z)\right)\right) d z \\
& +\int_{m_{i+1}}^{n_{i+1}} u\left(w_{0}-Q-K\left(m_{i+1}\right)\right) G^{\prime}(z) \\
& +\lambda\left(K\left(m_{i+1}\right)-C\left(F_{x}^{-1}(z)-K\left(m_{i+1}\right)\right)\right) d z \\
& +\lambda(Q-E x) \\
& =\int_{n_{i}}^{m_{i+1}} u\left(w_{0}-Q-K(z)\right) G^{\prime}(z) d z \\
& +\int_{n_{i}}^{m_{i+1}} \lambda K(z) d z \\
& -\int_{n_{i}}^{m_{i+1}} C\left(F_{x}^{-1}(z)-K(z)\right) d z
\end{aligned}
$$


$+\int_{m_{i+1}}^{n_{i+1}} u\left(w_{0}-Q-K\left(m_{i+1}\right)\right) G^{\prime}(z) d z$

$+\int_{m_{i+1}}^{n_{i+1}} \lambda K\left(m_{i+1}\right) d z$

$-\int_{m_{i+1}}^{n_{i+1}} C\left(F_{x}^{-1}(z)-K\left(m_{i+1}\right)\right) d z+\lambda(Q$

$-E x)$.

Let

$$
\begin{aligned}
I & =\int_{n_{i}}^{m_{i+1}} u\left(w_{0}-Q-K(z)\right) G^{\prime}(z) d z, \\
I I & =\int_{n_{i}}^{m_{i+1}} \lambda K(z) d z, \\
I I I & =-\int_{n_{i}}^{m_{i+1}} C\left(F_{x}^{-1}(z)-K(z)\right) d z, \\
I V & =\int_{m_{i+1}}^{n_{i+1}} u\left(w_{0}-Q-K\left(m_{i+1}\right)\right) G^{\prime}(z) d z, \\
V & =\int_{m_{i+1}}^{n_{i+1}} \lambda K\left(m_{i+1}\right) d z, \\
V I & =-\int_{m_{i+1}}^{n_{i+1}} C\left(F_{x}^{-1}(z)-K\left(m_{i+1}\right)\right) d z .
\end{aligned}
$$

Then,

$$
\begin{aligned}
U_{\lambda} & \left(K_{m_{i+1}}(z), z\right)=I+I I+I I I+I V+V+V I \\
& +\lambda(Q-E x), \\
I= & \int_{n_{i}}^{m_{i+1}} u\left(w_{0}-Q-K(z)\right) G^{\prime}(z) d z \\
& =-\int_{n_{i}}^{m_{i+1}} u\left(w_{0}-Q-K(z)\right) d\left(G\left(n_{i+1}\right)\right. \\
& -G(z)) \\
= & \int_{n_{i}}^{m_{i+1}}\left(G\left(n_{i+1}\right)-G(z)\right) d u\left(w_{0}-Q-K(z)\right) \\
& -\left.u\left(w_{0}-Q-K(z)\right)\left(G\left(n_{i+1}\right)-G(z)\right)\right|_{n_{i}} ^{m_{i+1}} \\
& =-\int_{n_{i}}^{m_{i+1}}\left(G\left(n_{i+1}\right)-G(z)\right) \\
& \cdot u^{\prime}\left(w_{0}-Q-K(z)\right) d K(z)-u\left(w_{0}-Q\right. \\
& \left.-K\left(m_{i+1}\right)\right)\left(G\left(n_{i+1}\right)-G\left(m_{i+1}\right)\right)+u\left(w_{0}-Q\right. \\
& \left.-K\left(n_{i}\right)\right)\left(G\left(n_{i+1}\right)-G\left(n_{i}\right)\right) .
\end{aligned}
$$

(D.1)

$$
\begin{aligned}
I V & =\int_{m_{i+1}}^{n_{i+1}} u\left(w_{0}-Q-K\left(m_{i+1}\right)\right) G^{\prime}(z) d z \\
& =\int_{m_{i+1}}^{n_{i+1}} u\left(w_{0}-Q-K\left(m_{i+1}\right)\right) d G(z)=u\left(w_{0}\right. \\
& \left.-Q-K\left(m_{i+1}\right)\right)\left(G\left(n_{i+1}\right)-G\left(m_{i+1}\right)\right) .
\end{aligned}
$$

So,

$$
\begin{aligned}
I+ & I V=-\int_{n_{i}}^{m_{i+1}}\left(G\left(n_{i+1}\right)-G(z)\right) \\
& \cdot u^{\prime}\left(w_{0}-Q-K(z)\right) d K(z)+u\left(w_{0}-Q\right. \\
& \left.-K\left(n_{i}\right)\right)\left(G\left(n_{i+1}\right)-G\left(n_{i}\right)\right) .
\end{aligned}
$$

Similarly,

$$
\begin{aligned}
I I & =\int_{n_{i}}^{m_{i+1}} \lambda K(z) d z \\
& =\left.\lambda K(z) z\right|_{n_{i}} ^{m_{i+1}}-\int_{n_{i}}^{m_{i+1}} \lambda z d K(z) \\
& =\lambda K\left(m_{i+1}\right) m_{i+1}-\lambda K\left(n_{i}\right) n_{i}-\int_{n_{i}}^{m_{i+1}} \lambda z d K(z) \\
V & =\int_{m_{i+1}}^{n_{i+1}} \lambda K\left(m_{i+1}\right) d z=\left.\lambda K\left(m_{i+1}\right) z\right|_{m_{i+1}} ^{n_{i+1}} \\
& =\lambda K\left(m_{i+1}\right)\left(n_{i+1}-m_{i+1}\right) .
\end{aligned}
$$

Hence,

$$
\begin{aligned}
I I+V= & -\lambda K\left(n_{i}\right) n_{i}-\int_{n_{i}}^{m_{i+1}} \lambda z d K(z) \\
& +\lambda K\left(m_{i+1}\right) n_{i+1}, \\
I I I= & -\int_{n_{i}}^{m_{i+1}} C\left(F_{x}^{-1}(z)-K(z)\right) d z \\
= & \int_{n_{i}}^{m_{i+1}} z d C\left(F_{x}^{-1}(z)-K(z)\right) \\
& -\left.C\left(F_{x}^{-1}(z)-K(z)\right) z\right|_{n_{i}} ^{m_{i+1}} \\
= & \int_{n_{i}}^{m_{i+1}} z C^{\prime}\left(F_{x}^{-1}(z)-K(z)\right) d F_{x}^{-1}(z) \\
& -\int_{n_{i}}^{m_{i+1}} z C^{\prime}\left(F_{x}^{-1}(z)-K(z)\right) d K(z) \\
& -C\left(F_{x}^{-1}\left(m_{i+1}\right)-K\left(m_{i+1}\right)\right) m_{i+1} \\
& +C\left(F_{x}^{-1}\left(n_{i}\right)-K\left(n_{i}\right)\right) n_{i},
\end{aligned}
$$




$$
\begin{aligned}
V I= & -\int_{m_{i+1}}^{n_{i+1}} C\left(F_{x}^{-1}(z)-K\left(m_{i+1}\right)\right) d z \\
= & \int_{m_{i+1}}^{n_{i+1}} z d C\left(F_{x}^{-1}(z)-K\left(m_{i+1}\right)\right) \\
& -\left.C\left(F_{x}^{-1}(z)-K\left(m_{i+1}\right)\right) z\right|_{m_{i+1}} ^{n_{i+1}} \\
= & \int_{m_{i+1}}^{n_{i+1}} z C^{\prime}\left(F_{x}^{-1}(z)-K\left(m_{i+1}\right)\right) d F_{x}^{-1}(z) \\
& -C\left(F_{x}^{-1}\left(n_{i+1}\right)-K\left(m_{i+1}\right)\right) n_{i+1} \\
& +C\left(F_{x}^{-1}\left(m_{i+1}\right)-K\left(m_{i+1}\right)\right) m_{i+1} .
\end{aligned}
$$

Thus,

$$
\begin{aligned}
I I I+ & V I \\
= & \int_{n_{i}}^{m_{i+1}} z C^{\prime}\left(F_{x}^{-1}(z)-K(z)\right) d F_{x}^{-1}(z) \\
& -\int_{n_{i}}^{m_{i+1}} z C^{\prime}\left(F_{x}^{-1}(z)-K(z)\right) d K(z) \\
& +C\left(F_{x}^{-1}\left(n_{i}\right)-K\left(n_{i}\right)\right) n_{i} \\
& +\int_{m_{i+1}}^{n_{i+1}} z C^{\prime}\left(F_{x}^{-1}(z)-K\left(m_{i+1}\right)\right) d F_{x}^{-1}(z) \\
& -C\left(F_{x}^{-1}\left(n_{i+1}\right)-K\left(m_{i+1}\right)\right) n_{i+1} .
\end{aligned}
$$

Then,

$$
\begin{aligned}
U_{\lambda} & \left(K_{m_{i+1}}(z), z\right) \\
& =-\int_{n_{i}}^{m_{i+1}}\left(G\left(n_{i+1}\right)-G(z)\right) u^{\prime}\left(w_{0}-Q-K(z)\right) \\
& +\lambda z+z C^{\prime}\left(F_{x}^{-1}(z)-K(z)\right) d K(z) \\
& +\int_{n_{i}}^{m_{i+1}} z C^{\prime}\left(F_{x}^{-1}(z)-K(z)\right) d F_{x}^{-1}(z) \\
& +\int_{m_{i+1}}^{n_{i+1}} z C^{\prime}\left(F_{x}^{-1}(z)-K\left(m_{i+1}\right)\right) d F_{x}^{-1}(z) \\
& -C\left(F_{x}^{-1}\left(n_{i+1}\right)-K\left(m_{i+1}\right)\right) n_{i+1}+u\left(w_{0}-Q\right. \\
& \left.-K\left(n_{i}\right)\right)\left(G\left(n_{i+1}\right)-G\left(n_{i}\right)\right)-\lambda K\left(n_{i}\right) n_{i} \\
& +\lambda K\left(m_{i+1}\right) n_{i+1}+C\left(F_{x}^{-1}\left(n_{i}\right)-K\left(n_{i}\right)\right) n_{i} \\
& +\lambda(Q-E x) .
\end{aligned}
$$

We plan to find $m_{i+1}^{*}$ such that

$$
U_{\lambda}\left(K_{m_{i+1}^{*}}(z), z\right)=\max _{n_{i}<m_{i+1} \leq n_{i+1}} U_{\lambda}\left(K_{m_{i+1}}(z), z\right)
$$

in order to obtain the global optimal solution.
It is easy to get

$$
\begin{aligned}
\frac{\partial U_{\lambda}}{}\left(K_{m_{i+1}}(z), z\right) & \partial m_{i+1} \\
= & -\left(G\left(n_{i+1}\right)-G\left(m_{i+1}\right)\right) u^{\prime}\left(w_{0}-Q-K\left(m_{i+1}\right)\right) \\
& -\lambda m_{i+1}-m_{i+1} C^{\prime}\left(F_{x}^{-1}\left(m_{i+1}\right)-K\left(m_{i+1}\right)\right) \\
& +m_{i+1} C^{\prime}\left(F_{x}^{-1}\left(m_{i+1}\right)-K\left(m_{i+1}\right)\right) \\
& -m_{i+1} C^{\prime}\left(F_{x}^{-1}\left(m_{i+1}\right)-K\left(m_{i+1}\right)\right) \\
= & -\left(G\left(n_{i+1}\right)-G\left(m_{i+1}\right)\right) u^{\prime}\left(w_{0}-Q-K\left(m_{i+1}\right)\right) \\
& -\lambda m_{i+1}-m_{i+1} C^{\prime}\left(F_{x}^{-1}\left(m_{i+1}\right)-K\left(m_{i+1}\right)\right) .
\end{aligned}
$$

Noticing $u^{\prime}(\cdot) \geq 0$ and $C^{\prime}(\cdot) \geq 0$, we can show $\partial U_{\lambda}\left(K_{m_{i+1}}(z)\right.$, $z) / \partial m_{i+1} \leq 0$. In other words, $U_{\lambda}\left(K_{m_{i+1}}(z), z\right)$ decreases on $\left[n_{i}, n_{i+1}\right]$. So, when $m_{i+1}=n_{i}$, we can get the maximal value of $U_{\lambda}\left(K_{m_{i+1}}(z), z\right)$. Therefore,

$$
K_{m_{i+1}}(z)=K_{n_{i}}(z)=K\left(n_{i}\right) I_{n_{i+1}>z>n_{i}} .
$$

It is not difficult to come to the conclusion that the optimal solution of Model 1 is as follows:

$$
\begin{aligned}
& I^{*}(x)=x-K\left(n_{i}\right) I_{n_{i+1}>z>n_{i}}, \\
& \qquad F_{x}^{-1}\left(n_{i+1}\right)>x>F_{x}^{-1}\left(n_{i}\right) .
\end{aligned}
$$

\section{Conflict of Interests}

The author declares that there is no conflict of interests regarding the publication of this paper.

\section{Acknowledgments}

The paper is supported by National Natural Science Foundation of China (71201051), the State Scholarship Fund (Grant no. 2014BQ11) and Young Talents Training Plan of Hunan Normal University (2014YX04), Philosophical and Social Science Fund of Hunan (no. 14YBA264), and Social Science Found of China (12\&ZD050 and 10BJL024).

\section{References}

[1] J. Mossin, "Aspects of rational insurance purchasing," Journal of Political Economy, vol. 76, no. 4, pp. 553-568, 1968.

[2] V. L. Smith, "Optimal insurance coverage," Journal of Political Economy, vol. 76, no. 1, pp. 68-77, 1968.

[3] J. P. Gould, "The expected utility hypothesis and the selection of optimal deductibles for a given insurance policy," The Journal of Business, vol. 42, no. 2, pp. 143-151, 1969.

[4] K. Borch, "The safety loading of reinsurancepremiums," Skandinavisk Aktuarietidskrift, pp. 84-162, 1960.

[5] K. J. Arrow, Essays in the Theory of Risk Bearing, Markham Publishing, Chicago, Ill, USA, 1971. 
[6] K. J. Arrow, Optimal Insurance and Generalized Deductibles, R-1 108-OEO, RAND Corporation, 1973.

[7] D. E. M. Sappington, "Incentives in principal-agent relationships," The Journal of Economic Perspectives, vol. 5, no. 2, pp. 45-66, 1991.

[8] J. von Neumann and O. Morgenstern, Theory of Games and Economic Behavior, Princeton University Press, Princeton, NJ, USA, 1944.

[9] A. Tversky and D. Kahneman, "Prospect theory: an analysis of decision under rick," Econometrica, vol. 47, no. 2, pp. 263-291, 1992.

[10] A. Tversky and D. Kahneman, "Advances in prospect theory: cumulative representation of uncertainty," Journal of Risk and Uncertainty, vol. 5, no. 4, pp. 297-323, 1992.

[11] J. Quiggin, "Comparative statics for rank-dependent expected utility theory," Journal of Risk and Uncertainty, vol. 4, no. 4, pp. 339-350, 1991.

[12] A. Chateauneuf, R.-A. Dana, and J.-M. Tallon, "Optimal risksharing rules and equilibria with Choquet-expected-utility," Journal of Mathematical Economics, vol. 34, no. 2, pp. 191-214, 2000.

[13] G. Carlier and R.-A. Dana, "Existence and monotonicity of solutions to moral hazard problems," Journal of Mathematical Economics, vol. 41, no. 7, pp. 826-843, 2005.

[14] G. Carlier and R.-A. Dana, "Rearrangement inequalities in nonconvex insurance models," Journal of Mathematical Economics, vol. 41, no. 4-5, pp. 483-503, 2005.

[15] R.-A. Dana and M. Scarsini, "Optimal risk sharing with background risk," Journal of Economic Theory, vol. 133, no. 1, pp. 152176, 2007.

[16] X. D. He and X. Y. Zhou, "Portfolio choice under cumulative prospect theory: an analytical treatment," Management Science, vol. 57, no. 2, pp. 315-331, 2011.

[17] X. D. He and X. Y. Zhou, "Portfolio choice via quantiles," Mathematical Finance, vol. 21, no. 2, pp. 203-231, 2011.

[18] G. Carlier and R. A. Dana, "Two-persons efficient risk-sharing and equilibria for concave law-invariant utilities," Economic Theory, vol. 36, no. 2, pp. 189-223, 2008.

[19] G. Carlier and R.-A. Dana, "Optimal demand for contingent claims when agents have law-invariant utilities," Mathematical Finance, vol. 21, no. 2, pp. 169-201, 2011.

[20] C. Bernard, X. D. He, J.-A. Yand, and X. Y. Zhou, "Optimal insurance design underrank-dependent expected utility", Mathematical Finance, vol. 25, no. 1, pp. 154-186, 2015.

[21] L. B. Dhiab, "Demand for insurance under rank dependent expected utility model," Research Journal of Finance and Accounting, vol. 6, no. 8, pp. 29-37, 2015.

[22] L. F. Ackert and R. Deaves, Behavioral Finance: Psychology, Decision-Making, and Markets, Cengage Learning, 2013.

[23] G. Choquet, “Theory of capacities," Annales de l'Institut Fourier, vol. 5, pp. 131-295, 1953.

[24] A. Raviv, "The design of an optimal insurance policy," The American Economic Reiew, vol. 69, no. 1, pp. 84-96, 1979.

[25] J. H. Abbring, P.-A. Chiappori, J. J. Heckman, and J. Pinquet, "Adverse selection and moral hazard in insurance: can dynamic data help to distinguish?" Journal of the European Economic Association, vol. 1, no. 2-3, pp. 512-521, 2003.

[26] P. Chiappori and B. Salani, "Empirical contract theory: the case of insurance data," European Economic Review, vol. 41, no. 3-5, pp. 943-950, 1997.
[27] M. Landsberger and I. Meilijson, "Extraction of surplus under adverse selection: the case of insurance markets," Journal of Economic Theory, vol. 69, no. 1, pp. 234-239, 1996.

[28] C. Bernard, X. He, J. Yan, and X. Y. Zhou, "Optimal insurance design under rank-dependent expected utility," Mathematical Finance, vol. 25, no. 1, pp. 154-186, 2015. 


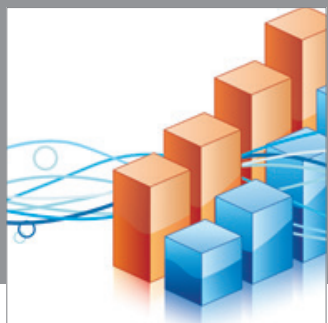

Advances in

Operations Research

mansans

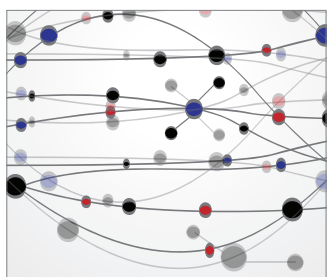

The Scientific World Journal
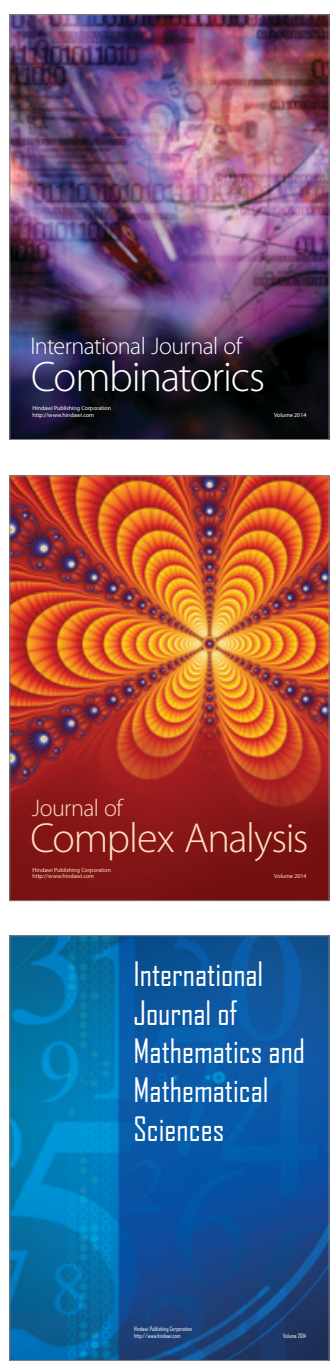
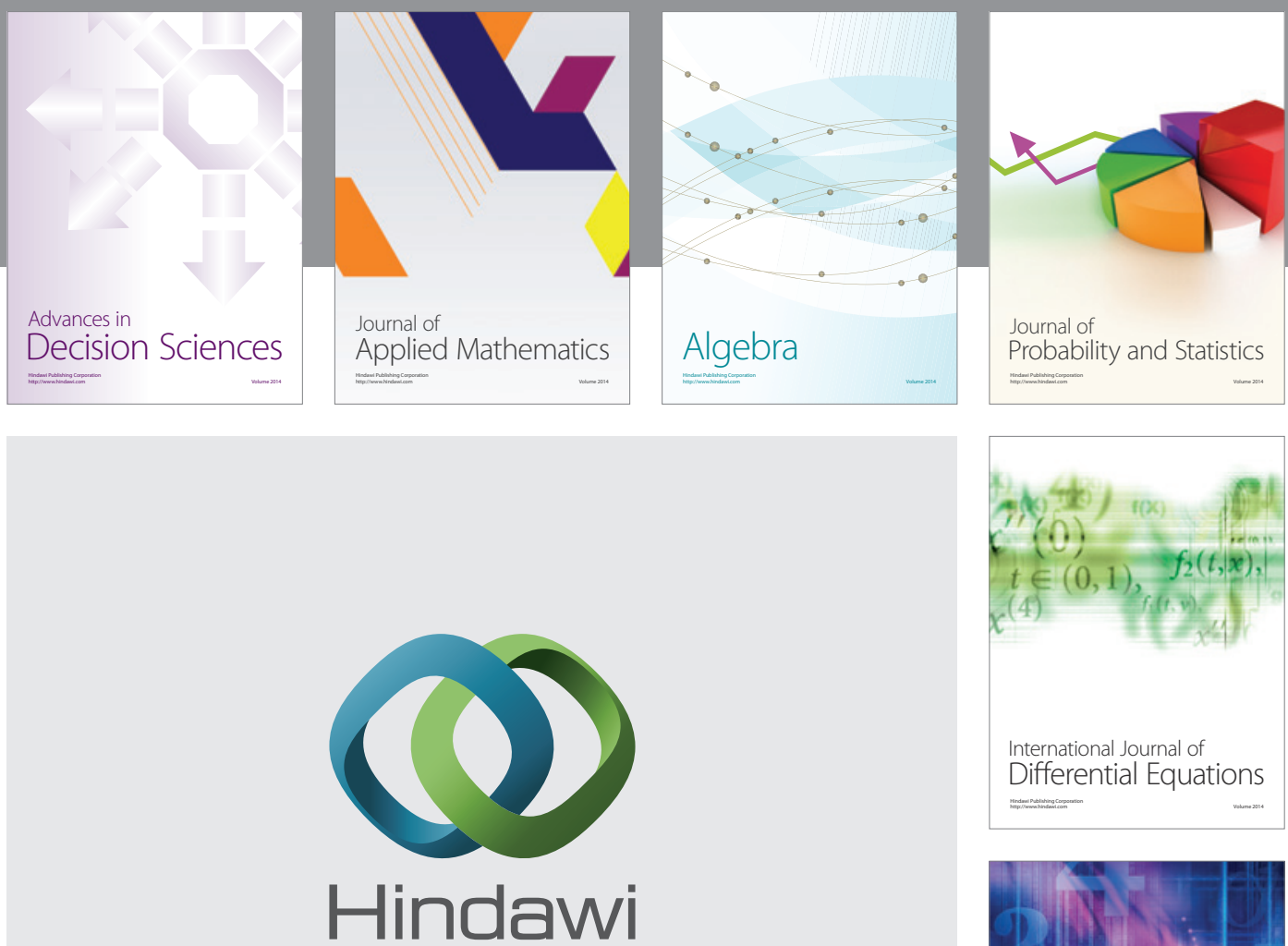

Submit your manuscripts at http://www.hindawi.com
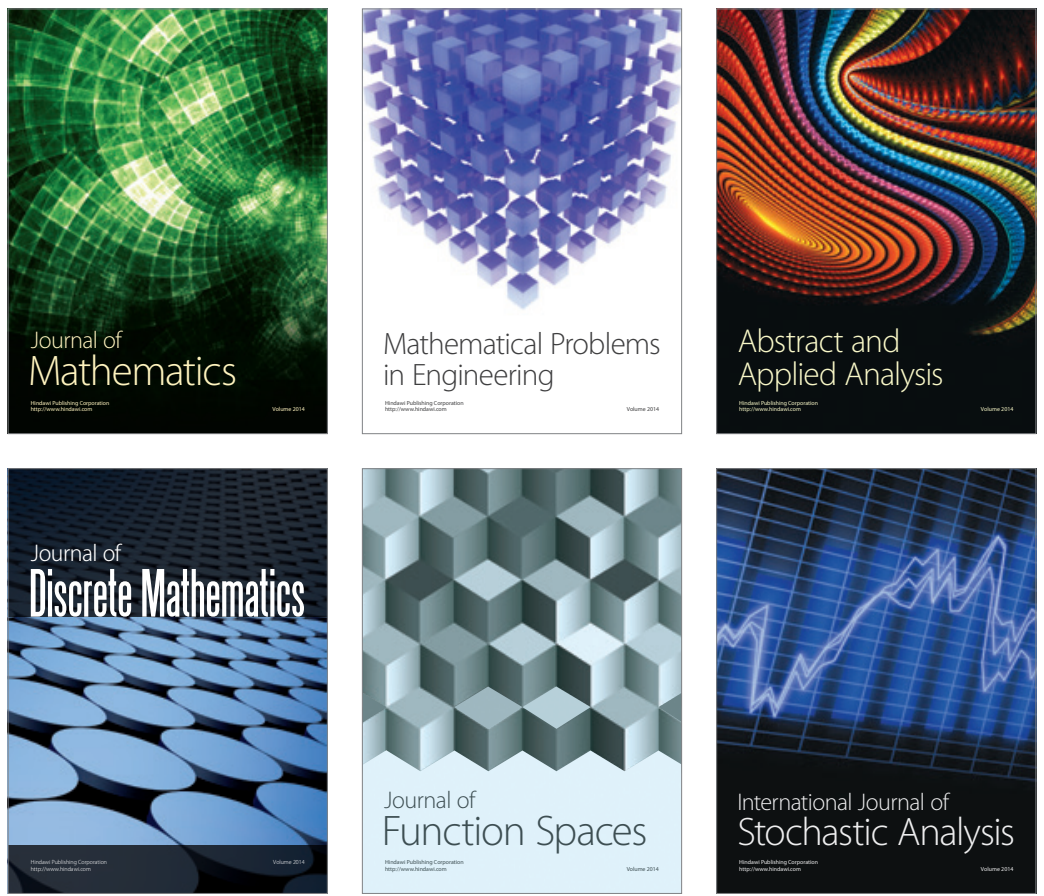

Journal of

Function Spaces

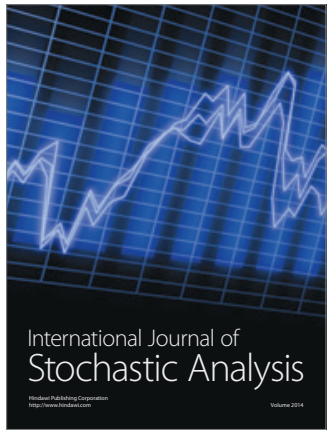

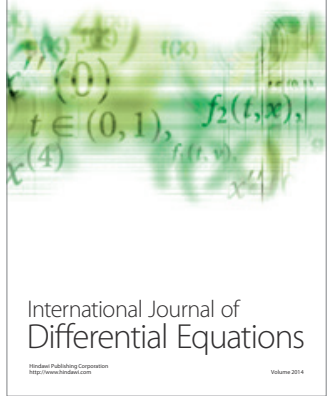
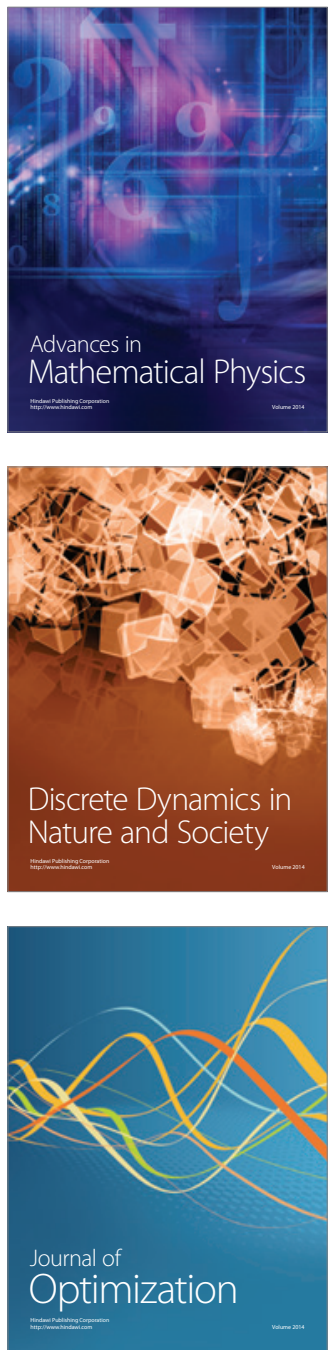